\title{
Performance of Akatsuki/IR2 in Venus orbit: the first year
}

\author{
Takehiko Satoh ${ }^{1,2^{*}}$, Takao M. Sato ${ }^{1}$, Masato Nakamura', Yasumasa Kasaba ${ }^{3}$, Munetaka Ueno ${ }^{4}$, Makoto Suzuki ${ }^{1}$, \\ George L. Hashimoto ${ }^{5}$, Takeshi Horinouchi ${ }^{6}$, Takeshi Imamura ${ }^{7}$, Atsushi Yamazaki ${ }^{1}$, Takayuki Enomoto ${ }^{8}$, \\ Yuri Sakurai ${ }^{9}$, Kosuke Takami ${ }^{3}$, Kenta Sawai ${ }^{9}$, Takashi Nakakushi ${ }^{10}$, Takumi Abe ${ }^{1}$, Nobuaki Ishii ${ }^{1}$, \\ Chikako Hirose ${ }^{1}$, Naru Hirata ${ }^{11}$, Manabu Yamada ${ }^{12}$, Shin-ya Murakami ${ }^{1}$, Yukio Yamamoto ${ }^{1}$, Tetsuya Fukuhara ${ }^{13}$, \\ Kazunori Ogohara ${ }^{14}$, Hiroki Ando ${ }^{15}$, Ko-ichiro Sugiyama ${ }^{16}$, Hiroki Kashimura ${ }^{4}$ and Shoko Ohtsuki ${ }^{17}$
}

\begin{abstract}
The first year (December 2015 to November 2016) of IR2 after Akatsuki's successful insertion to an elongated elliptical orbit around Venus is reported with performance evaluation and results of data acquisition. The single-stage Stirlingcycle cryo-cooler of IR2 has been operated with various driving voltages to achieve the best possible cooling under the given thermal environment. A total of 3091 images of Venus (1420 dayside images at $2.02 \mu \mathrm{m}$ and 1671 night-side images at 1.735, 2.26, and $2.32 \mu \mathrm{m}$ ) were acquired in this period. Additionally, 159 images, including images of stars for calibration and dark images for the evaluation of noise levels, were captured. Low-frequency flat images (not available in pre-launch calibration data) have been constructed using the images of Venus acquired from near the pericenter to establish the procedure to correct for the IR2 flat-field response. It was noticed that multiple reflections of infrared light in the PtSi detector caused a weak but extended tail of the point-spread function (PSF), contaminating the night-side disk of Venus with light from the much brighter dayside crescent. This necessitated the construction of an empirical PSF to remove this contamination and also to improve the dayside data by deconvolution, and this work is also discussed. Detailed astrometry is performed on star-field images in the $\mathrm{H}$-band $(1.65 \mu \mathrm{m})$, hereby confirming that the geometrical distortion of IR2 images is negligible.
\end{abstract}

Keywords: Venus, Atmosphere, Orbiter, Akatsuki, Near-infrared, Window, Cryo-cooler, Dynamics, Aerosols

\section{Introduction}

Akatsuki, also known as the Japanese Venus Climate Orbiter (Nakamura et al. 2011), arrived at Venus in December 2015, approximately a year after the end of the European Venus Express (VEx; Svedhem et al. 2009; Titov et al. 2009) mission, thus enabling a nearly continuous history of recording Earth's sister planet. In contrast to the elongated polar orbit of VEx, Akatsuki was inserted into an elongated and retrograde (westward) orbit near Venus's equatorial plane, best suited to study atmospheric dynamics, the super rotation in particular,

\footnotetext{
${ }^{*}$ Correspondence: satoh@stp.isas.jaxa.jp

${ }^{1}$ Institute of Space and Astronautical Science, Japan Aerospace Exploration Agency, 3-1-1 Yoshinodai, Chuo-ku, Sagamihara, Kanagawa 252-5210, Japan

Full list of author information is available at the end of the article
}

by intensively observing the equatorial to middle-latitude regions (Nakamura et al. 2016).

IR2, a cryo-cooled 2- $\mu \mathrm{m}$ camera, one of six scientific instruments onboard Akatsuki, shares with VEx/VIRTIS (Visible, Infrared and Thermal Imaging Spectrometer; Drossart et al. 2007; Piccioni et al. 2007) the strategy to probe the middle-to-lower atmosphere of Venus by utilizing near-infrared (NIR) "windows" of the $\mathrm{CO}_{2}$ atmosphere (Allen and Crawford 1984; Taylor and Crisp 1997; Peralta et al. 2017). On IR2, these observations are made possible by a set of narrow-band filters $(1.735,2.26$, and $2.32 \mu \mathrm{m}$ ) installed in a 6-position filter wheel (Satoh et al. 2016; hereafter referred to as Paper I). The scientific objectives with these filters include studying the atmospheric dynamics by measuring the cloud motion vectors (Hueso et al. 2012), morphology of clouds (McGouldrick 
et al. 2008), aerosol properties (Carlson et al. 1993; Haus et al. 2015) by combining 1.735 - and $2.26-\mu \mathrm{m}$ images, and mapping the sub-cloud CO (at $\sim 35 \mathrm{~km}$ altitudes) by differentiating the 2.26- and 2.32- $\mu \mathrm{m}$ quasi-simultaneous image pair.

In addition, IR2 is equipped with a $2.02-\mu \mathrm{m}$ filter which is used to acquire images of the dayside disk of Venus in the strong $\mathrm{CO}_{2}$ absorption band. This band is particularly useful to infer the cloud-top altitude as was previously done in a different $\mathrm{CO}_{2}$ absorption band $(1.6 \mu \mathrm{m})$ with VEx/VIRTIS (Ignatiev et al. 2009).

As with many other space instruments, IR2, placed in Venus orbit, experienced several problems that need to be addressed before observations can be regularly conducted and the data stably produced. The thermal condition of the spacecraft is a key issue for IR2, as the compressor and cold head of the cooling system, mounted on the radiator plate, are exposed to space (Paper I) and therefore are subject to solar illumination (Fig. 1). In this paper, we describe the operation of IR2 and the results obtained during its first year of orbiting Venus, from December 2015 to November 2016, including the problems that have arisen, how we overcome them, and the quality of IR2 data after these issues were solved. The scientific results, which require detailed analyses, will be reported in subsequent papers.

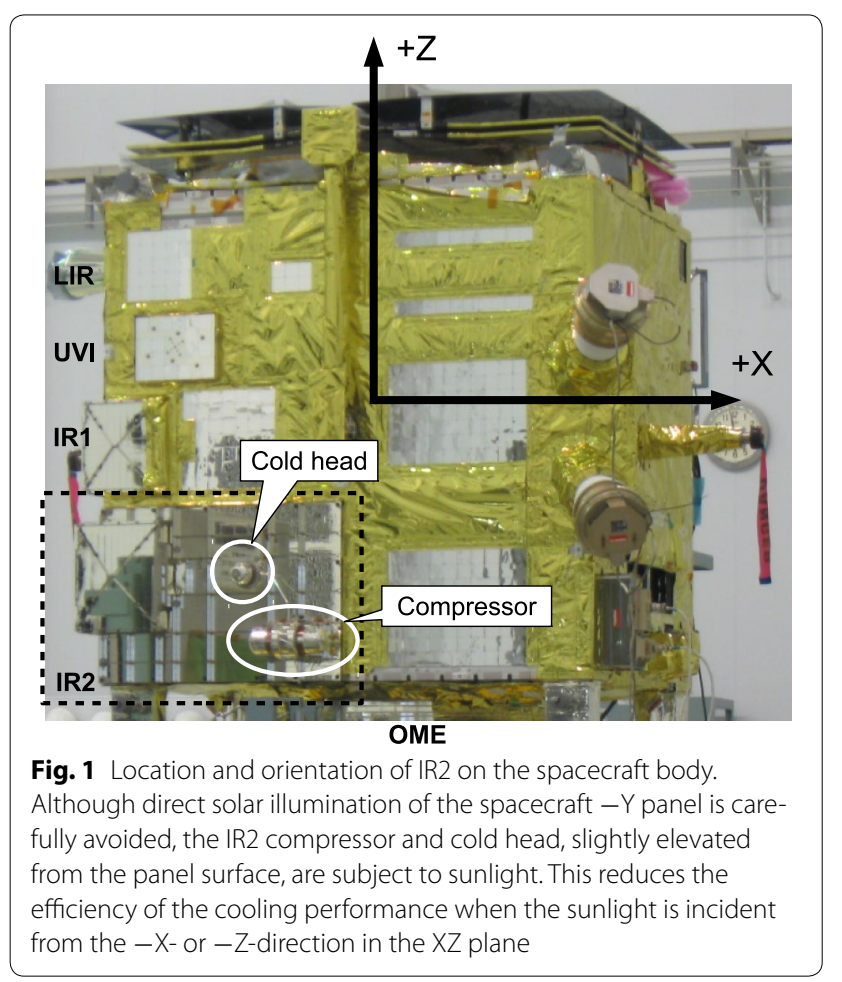

\section{Operation of IR2 cryo-cooler and thermal history Overview}

Cooling of the PtSi detector $(<70 \mathrm{~K})$ with a single-stage Stirling-cycle cryo-cooler is essential to Venus observations with IR2 as thermally produced electrons would saturate the detector in a few tens of seconds when the detector temperature is $70 \mathrm{~K}$ (Paper I). The cooling power is controlled by the input voltage (a sinusoidal wave of $50-\mathrm{Hz}$ frequency) from the driver electronics (IR2-CDE) to the compressor of the cooler. The IR2-CDE measures the current (STC_I_OP) and then computes the effective power (STC_P_OP), corresponding to the input voltage (STC_V_SET). Because of the internal mechanism of the cryo-cooler, there is no simple conversion from STC_V SET to STC_P_OP; instead, the actual measurement of STC_I_OP is necessary. The rules of IR2 cooler operation are to start with a low voltage $(10.61 \mathrm{~V})$ to smoothen the motion of the internal mechanism of the cooler. After achieving the cold-chip temperature of $<180 \mathrm{~K}$, the input voltage can gradually be increased. The nominal driving voltage is $17.24 \mathrm{~V}$, which is expected to achieve an effective cooling power of $50 \mathrm{~W}$.

On December 10, 2015, three days after the success of VOI-R1 (Venus orbit insertion-revenge 1; Nakamura et al. 2016), the IR2 cryo-cooler was started. The operation of the cryo-cooler in the period from December 2015 to November 2016 is summarized in Table 1. The time history of the driving voltage (STC_V_SET), the current (STC_I_OP), the effective power (STC_P_OP), and the detector temperature is plotted in Fig. 2. In addition, Fig. 2 also shows the observations of Venus with IR2 (yellow and navy triangles for the dayside and nightside observations, respectively). Since the cooling of IR2 is achieved by maintaining a delicate balance between the incoming heat and the removal of heat by the cryocooler, the detector temperature is affected by the solar illumination condition of IR2. Details are discussed in "Cooling performance (sun-angle dependence)" section.

\section{Unexpected shutdowns of the cooler (January 2016)}

There were two unexpected shutdowns of the IR2 cryocooler, of which the first happened when the spacecraft was about to exit from the penumbra (January 20, 2016). The exact cause thereof has not been identified, but it is now known that the voltage control capability of IR2$\mathrm{CDE}$ is temperature dependent. At low temperatures (below approximately $15^{\circ} \mathrm{C}$ ), the electric circuit of IR2$\mathrm{CDE}$ is unable to supply sufficient current to the compressor, causing the sinusoidal wave to become distorted at the peaks. Evidence suggests that this is caused by insufficient electric charge on the capacitor in IR2-CDE, 
Table 1 Operation of IR2 cryo-cooler

\begin{tabular}{|c|c|c|c|c|c|}
\hline ID & Date (UT) & Event & $V(\mathrm{~V})$ & $P(W)$ & Remarks \\
\hline \multirow[t]{2}{*}{1} & $12 / 10 / 2015$ & Start the cooler & 10.61 & 15 & \\
\hline & $12 / 10$ & Increase the driving voltage & 13.25 & 30 & $16 \mathrm{~h}$ after start-up \\
\hline 2 & $12 / 11$ & First 2.02- $\mu \mathrm{m}$ image & 13.25 & 30 & \\
\hline 3 & $01 / 20 / 2016$ & Unexpected shutdown & 13.25 & 30 & Before the end of penumbra \\
\hline \multirow[t]{2}{*}{4} & $01 / 25$ & Restart the cooler & 10.61 & 15 & \\
\hline & $01 / 26$ & Increase the driving voltage & 13.25 & 30 & \\
\hline 5 & $01 / 28$ & Unexpected shutdown & 16.08 & N/A & Large fluctuation in current \\
\hline \multirow[t]{2}{*}{6} & $02 / 12$ & No cooling when restarted & 10.61 & 15 & \\
\hline & 03/09 & External heater to warm up & - & - & \\
\hline \multirow[t]{2}{*}{7} & $03 / 10$ & Restart the cooler & 10.61 & 15 & Successfully restarted \\
\hline & $03 / 11$ & Increase the driving voltage & 13.25 & 30 & \\
\hline 8 & $03 / 15$ & Planned shutdown & - & - & To remove contaminants \\
\hline 9 & $03 / 15-03 / 21$ & External heater to warm up & - & - & \\
\hline \multirow[t]{2}{*}{10} & $03 / 22$ & Restart the cooler & 10.61 & 15 & Successfully restarted \\
\hline & $03 / 22$ & Increase the driving voltage & 15.25 & 40 & Via 13.25 V (30 W) \\
\hline \multirow[t]{3}{*}{11} & $04 / 18-04 / 22$ & Testing small-step increases & & & Small UPs and DOWNs \\
\hline & $04 / 28$ & Increase the driving voltage & 15.92 & & Via $15.59 \mathrm{~V}$ \\
\hline & $04 / 29$ & Increase the driving voltage & 16.08 & & \\
\hline 12 & $04 / 30$ & Increase the driving voltage & 16.25 & 46 & This became the "nominal" \\
\hline 13 & $05 / 27$ & Enter the superior conj. mode & 13.25 & 30 & No obs. during superior conj. \\
\hline \multirow[t]{7}{*}{14} & $06 / 16$ & Recover from superior conj. mode & 13.60 & & \\
\hline & $06 / 17$ & Recover from superior conj. mode & 14.26 & & Via $13.93 \mathrm{~V}$ \\
\hline & $06 / 20$ & Recover from superior conj. mode & 14.92 & & Via $14.59 \mathrm{~V}$ \\
\hline & $06 / 21$ & Recover from superior conj. mode & 15.25 & 40 & \\
\hline & $06 / 23$ & Recover from superior conj. mode & 15.59 & & \\
\hline & $06 / 24$ & Recover from superior conj. mode & 15.92 & & \\
\hline & $06 / 27$ & Recover from superior conj. mode & 16.08 & & \\
\hline 15 & $06 / 28$ & Recover from superior conj. mode & 16.25 & 46 & \\
\hline 16 & $11 / 07$ & Decrease the driving voltage & 15.25 & & Attempt to improve stability \\
\hline
\end{tabular}

while the pulse-width modulation (PWM) cycle is in idle mode and/or decreased gain of the output FET at low temperatures. When the sinusoidal wave becomes distorted, STC_P_OP drops (often discontinuously) and the power consumption of IR2-CDE fluctuates. Although the exact same symptom has not been reproduced by the ground model, this may likely be related to the unexpected shutdown of the cooler.

The second shutdown happened on January 28, 2016. The cryo-cooler was restarted on the $25 \mathrm{th}$, after the shutdown of the 20th, with a low driving voltage $(10.61 \mathrm{~V})$. On the 26th, we increased the driving voltage to $13.25 \mathrm{~V}$ with success (approximately $30 \mathrm{~W}$ of effective power). On the 28th, however, shutdown occurred when the voltage reached $16.08 \mathrm{~V}$ while targeting $16.24 \mathrm{~V}(\sim 45 \mathrm{~W})$. The telemetry indicated that unstable driving was noticed when the voltage was $15.92 \mathrm{~V}$ and the excessively large fluctuation of STC_I_OP at $16.08 \mathrm{~V}$ may have triggered the shutdown (built-in protection in the power supply unit). Later, the cause of the unexpected shutdown was suspected to be the accumulation of contaminants in the cooler that may have prevented smooth motion of the moving parts.

\section{No cooling problem (February 2016)}

After the second shutdown (January 28, 2016), we attempted to restart the cooler by following the usual procedure (10.61-V driving voltage) on February 12, 2016. After monitoring the telemetry for over an hour, however, it was noticed that the cold-chip temperature did not fall. (The expectation was to fall by at least several digits.) The most likely cause thereof is the presence of frozen contaminants in the compressor or in the cold head. After the second shutdown, these components were mostly below $-10{ }^{\circ} \mathrm{C}$ which is low enough to allow water vapor (if the partial pressure is $\sim 100 \mathrm{ppmv}$ or more) to freeze inside the cooler. Therefore, the moving parts were unable to move when a driving power of 

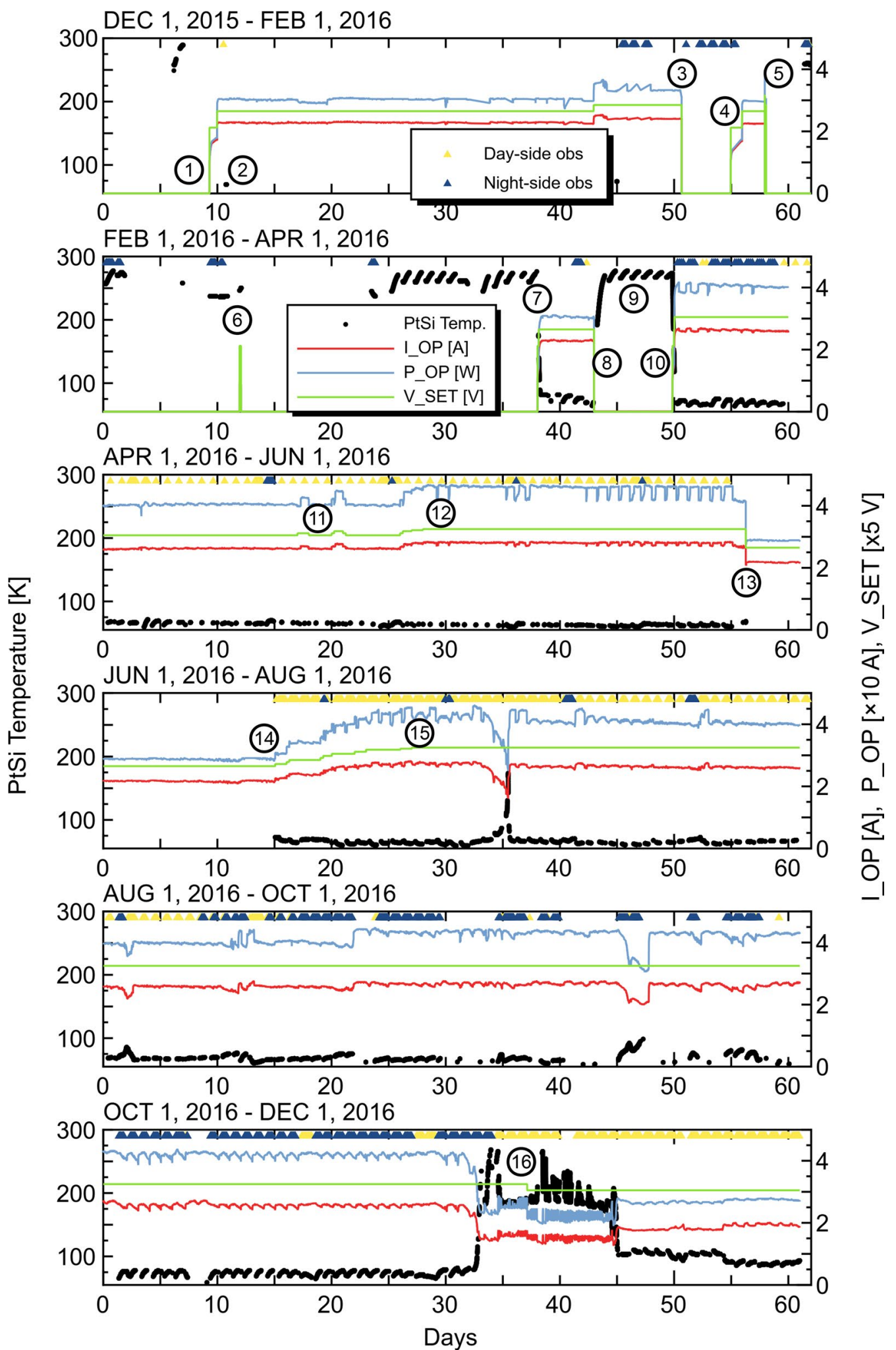

Fig. 2 History of IR2 cryo-cooler operation and the PtSi detector temperature 
$10.61 \mathrm{~V}$ was enforced. We solved this problem by changing the threshold temperature of the external heater and used solar illumination (Fig. 1) as well to maintain these components at temperatures above $0{ }^{\circ} \mathrm{C}$, the melting point of contaminants. This operation to remove the contaminants took place for a week from March 15 to 22, 2016. Subsequently, the cooler was successfully started and a driving voltage of $15.25 \mathrm{~V}(\sim 40 \mathrm{~W})$ was applied.

\section{Normal operation and lessons learned}

The driving voltage was maintained at $15.25 \mathrm{~V}$ until the last week of April 2016, to ensure the cooler does not become unstable during umbra passages (March 25 and April 4) and the orbit correction maneuver (April 4). Considering the shutdown incident of January 28, we avoided increasing the applied voltage suddenly from $15.25-\mathrm{V}$ drive to $17.25-\mathrm{V}$ drive but decided to apply a voltage between these two values, $16.25 \mathrm{~V}$. From April 18 to 22 , we practiced increasing and decreasing the voltage in very small steps (a few tens of volts) using UPs and DOWNs of the driving voltage to ensure these small steps would not destabilize the cooler. After these practice runs and another umbra passage (April 26), the driving voltage applied to the cooler was increased in small steps on April 28, 29, and 30, until the final driving voltage of $16.25 \mathrm{~V}(\sim 46 \mathrm{~W})$ was achieved.

At the time Akatsuki arrived at Venus, approximately 6 years had passed since the cryo-cooler was charged with pressurized helium gas; thus, contamination due to outgassing was almost unavoidable. $\left(\mathrm{H}_{2} \mathrm{O}\right.$ and $\mathrm{CO}_{2}$ are the major outgassing molecules from metal surface inside the cryo-cooler.) The problems that were experienced, partially if not all, may have been caused by this and would definitely continue to prevail. Therefore, the practice runs from January to April 2016 were valuable as we learned appropriate ways to operate the "aged" cryo-cooler to achieve sufficient cooling of the IR2 components.

\section{"Superior conjunction" mode}

On June 7, 2016, Venus as viewed from the earth disappeared behind the sun (superior conjunction). The resulting limited telecommunication (no command uplink when the spacecraft in the sky is within $2.5^{\circ}$ from the sun) prevented Venus from being observed or data from being received during this period (the so-called superior conjunction mode). In anticipation, the driving power of IR2 was lowered to $13.25 \mathrm{~V}$ on May 27 to avoid unnecessary wear. When communication with the spacecraft resumed, the driving power of IR2 was again raised to the pre-conjunction value $(16.25 \mathrm{~V})$. As summarized in Table 1 , the recovery process required almost 2 weeks, but, due to very careful power control, the cooler did not indicate instability. The nominal driving power (16.25 V) was achieved again on June 28, 2016.

\section{Cooling performance (sun-angle dependence)}

Successful cooling of IR2 depends on achieving a balance between the incoming heat and the removal of heat by the cryo-cooler. The former increases when sunlight illuminates the $-\mathrm{X}$-panel of the spacecraft (where the camera bore is located) and the $-\mathrm{Z}$-panel (where the thruster of the orbital-maneuver engine is located), causing higher detector temperature than is usable. This situation happens when the night-side of Venus is observed (indicated with navy triangles in Fig. 2). Therefore, images of the night-side of Venus tend to undergo gradual degradation after a few hours (up to 4 or $6 \mathrm{~h}$ may be acceptable for the solar phase angles $\sim 150^{\circ}$ ), thereby to some extent limiting the cloud-tracking on the night-side at very large solar phase angles.

\section{Summary of first-year observations Observation programs and exposure times}

Akatsuki's scientific observations are conducted with a set of so-called observation programs. At each step of an observation program, a "sensor control" command (TAKE IMAGE, for example) and an "image operation" command (IMAGE_SUB, for example) can be performed in parallel. The observation programs relevant to IR2 are summarized in Table 2. Note that observation programs 0x0D, 0x0E, $0 x 17$, and $0 x 1 F$ utilize "region of interest (ROI)" function with which a "user-defined" sub-frame is stored on the data recorder, thus saving storage space and downlink time.

Since IR2 does not have a mechanical shutter and its detector does not have frame-transfer architecture, the exposure time is the sum of the interval between "pre-" and "post"-readouts (wipes) plus one readout time. The shortest possible exposure time (6.97 s) is therefore the readout time (zero interval) mostly limited by the speed of the 14-bit AD converter. The permitted length of an interval is programmed in a so-called clock pattern that performs "pre-" and "post"-readouts and waits for the preset interval. There are several clock patterns with different intervals (multiples of $6 \mathrm{~s}$ ), and the choice is prescribed in the observation program. (Different clock patterns may be called for different filters in one observation program.) The exposure times provided in Table 2 are thus defined by the choice of clock patterns.

\section{Total volume of acquired data}

The number of images for each of the four filters (2.02, $1.735,2.26$, and $2.32 \mu \mathrm{m}$ ), acquired during the first year in orbit (December 2015 to November 2016), is summarized in Table 3. 
Table 2 Observation programs (only IR2 relevant)

\begin{tabular}{|c|c|c|c|c|c|}
\hline No. (Hex) & Name & $2.02 \mu \mathrm{m}$ & $1.735 \mu \mathrm{m}$ & $2.26 \mu \mathrm{m}$ & $2.32 \mu \mathrm{m}$ \\
\hline $01(0 \mathrm{D})^{\mathrm{a}}$ & Night deluxe & $12.97 \mathrm{~s}(3 \mathrm{~m})^{\mathrm{b}}$ & $6.97 \mathrm{~s}(3 \mathrm{~m})^{\mathrm{b}}$ & $6.97 \mathrm{~s}(3 \mathrm{~m})^{\mathrm{b}}$ & $12.97 \mathrm{~s}(3 \mathrm{~m})^{\mathrm{b}}$ \\
\hline $02(0 \mathrm{E})^{\mathrm{a}}$ & Night slim & & & $6.97 \mathrm{~s}(3 \mathrm{~m})^{\mathrm{b}}$ & \\
\hline $05(17)^{\mathrm{a}}$ & Day deluxe & $6.97 \mathrm{~s}(3 \mathrm{~m})^{\mathrm{b}}$ & & & \\
\hline $06(1 F)^{a}$ & Day slim & $6.97 \mathrm{~s}(3 \mathrm{~m})^{\mathrm{b}}$ & & & \\
\hline$O A$ & Limb & $18.97 \mathrm{~s}$ & & & \\
\hline$O B$ & Night pericenter scan & & $6.97 \mathrm{~s}$ & $6.97 \mathrm{~s}$ & \\
\hline$O C$ & Day pericenter scan & $6.97 \mathrm{~s}$ & & & \\
\hline 12 & IR2 star observation & & $18.97 \mathrm{~s}$ & $12.97 \mathrm{~s}$ & $18.97 \mathrm{~s}$ \\
\hline 14 & IR2 health check & $6.97 \mathrm{~s}$ & & & \\
\hline
\end{tabular}

${ }^{a}$ Numbers in parentheses are the observation programs with "region of interest (ROI)" function enabled

b $(3 \mathrm{~m})$ indicates the acquired image is the median of 3 images (processed onboard)

Table 3 Summary of acquired images (December 2015-November 2016)

\begin{tabular}{|c|c|c|c|c|c|c|c|c|c|c|c|c|}
\hline \multirow[t]{2}{*}{ Filter $(\mu \mathrm{m})$} & \multicolumn{10}{|c|}{ Venus (by observation programs) } & \multirow{2}{*}{$\begin{array}{l}\text { Others } \\
\text { Star }\end{array}$} & \multirow[t]{2}{*}{ Total } \\
\hline & $01(0 D)$ & $02(0 \mathrm{E})$ & $05(17)$ & $06(1 F)$ & $O A$ & $\mathrm{OB}$ & OC & 12 & 14 & Total & & \\
\hline 1.735 & 549 & N/A & N/A & N/A & N/A & 8 & N/A & 3 & N/A & 560 & 30 & 590 \\
\hline 2.26 & 548 & 0 & N/A & N/A & N/A & 8 & N/A & 3 & N/A & 559 & 30 & 589 \\
\hline 2.32 & 549 & N/A & N/A & N/A & N/A & N/A & N/A & 3 & N/A & 552 & 30 & 582 \\
\hline 2.02 & 450 & N/A & 739 & 193 & 2 & N/A & 12 & N/A & 24 & 1420 & 16 & 1436 \\
\hline Dark & & & & & & & & & & 53 & Grand total & 3250 \\
\hline
\end{tabular}

Examples of images of Venus (dayside and night-side) are displayed in Fig. 3. These images are "dark subtracted (onboard)," "flat-field corrected (discussed in 5.1 and 5.2)," and "brightness of quadrants adjusted (discussed in 5.3)." Note that although these processes improve the scientific usefulness of IR2 images, they do not significantly change their appearance.

The quality of the data is evaluated in "Quality of the data" section for performance on fine-scale cloud features and photometric accuracy.

\section{Evaluation of dark noise}

Every time IR2 observes Venus; dark frames are acquired before and after the image of Venus is captured. These two dark frames are averaged and subtracted onboard from the raw images of Venus. The dark frames are stored in a dedicated but smaller and overwrite-enabled partition on the data recorder and are usually not replayed during the downlink to the ground. We evaluated the dark noise as a function of detector temperature by downlinking the IR2 dark frames for three occasions: on October 26, 2010, when images of the earth and the moon were acquired; on February 28, 2011, at the beginning of full-disk photometry of Venus (Satoh et al. 2015); and most intensively during the period May 10-24, 2016 (after the VOI-R), to characterize the instrument right before the superior conjunction.
Since the detector temperature during the period in May 2016 was the lowest ( $61.85 \mathrm{~K}$ ) on the 22nd, the dark image acquired on May-22 is used as the reference and is subtracted from the data of the other dates. This is necessary as there are slight offsets of the zero levels (varying from one quadrant to another) that need to be canceled by using this subtraction. Then, according to Richardson's equation (see, e.g., Wigner 1997) with a barrier height of $0.1985 \mathrm{eV}$ (slightly adjusted from $0.197 \mathrm{eV}$ in Paper I to more accurately reproduce the high noise counts in February 2011), we specified an offset of 2300 electrons (expected for a pixel of $61.85 \mathrm{~K}$ in 6.97-s integration) for all data. The relation of the obtained dark noise versus temperature is plotted in Fig. 4. The overall behavior of the measurement points is consistent with the theoretical curve. This offers a plausible explanation that the detector becomes saturated by thermal electrons when the temperature exceeds $73 \mathrm{~K}$.

\section{Validation of absolute calibration: photometry of Antares images}

The images of Antares ( $\alpha$ Scorpii) were acquired on September 8,2016 , with four filters $(2.02,1.735,2.26$, and $2.32 \mu \mathrm{m})$. The observation program $0 \times 12$ was executed five times such that the exposure times for the three night filters are $18.97 \mathrm{~s}$ for $1.735 \mu \mathrm{m}, 12.97 \mathrm{~s}$ for $2.26 \mu \mathrm{m}$, and $18.97 \mathrm{~s}$ for $2.32 \mu \mathrm{m}$, respectively (see Table 2). For 


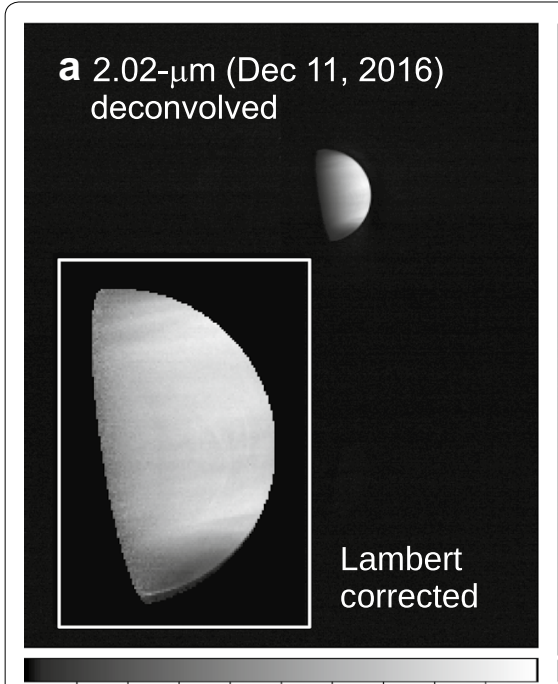

$\begin{array}{lllllllll}0.48 & 0.98 & 1.5 & 2 & 2.5 & 3 & 3.5 & 4 & 4.5\end{array}$

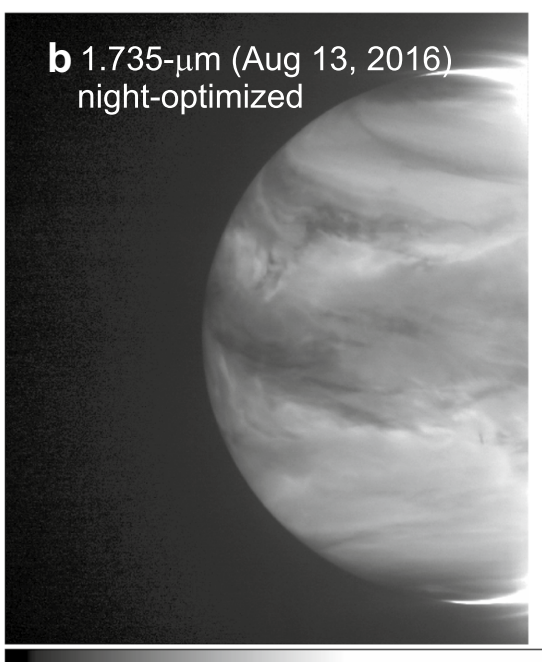

$\begin{array}{lllllllll}0.029 & 0.059 & 0.089 & 0.12 & 0.15 & 0.18 & 0.21 & 0.24 & 0.27\end{array}$ Radiance [W m ${ }^{-2} \mathrm{sr}^{-1} \mu \mathrm{m}^{-1}$ ]

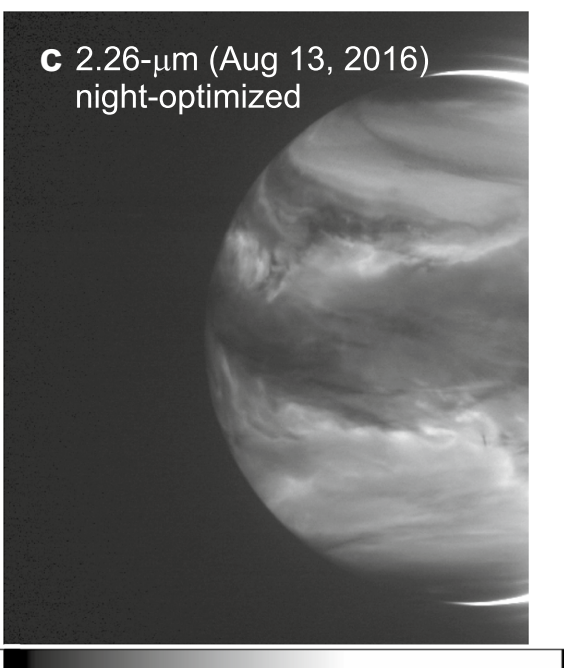

$\begin{array}{lllllllll}0.019 & 0.039 & 0.059 & 0.08 & 0.1 & 0.12 & 0.14 & 0.16 & 0.18\end{array}$

Fig. 3 Example of IR2 images obtained during Venus orbit. a First-light Venus image acquired with IR2 at 2.02 um (December 11, 2015, deconvolved with the PSF described in the text). The inset is corrected for limb darkening by assuming Lambertian intensity distribution. The large-scale bow-shaped feature is hinted at although not as prominent as in LIR images (Fukuhara et al. 2017); b, c example images of the night-side of Venus at 1.735 and $2.26 \mu \mathrm{m}$ (August 13, 2016, acquired with "night-optimized" observation). The 2.32- $\mu \mathrm{m}$ image is omitted as it appears almost identical to the $2.26-\mu \mathrm{m}$ image

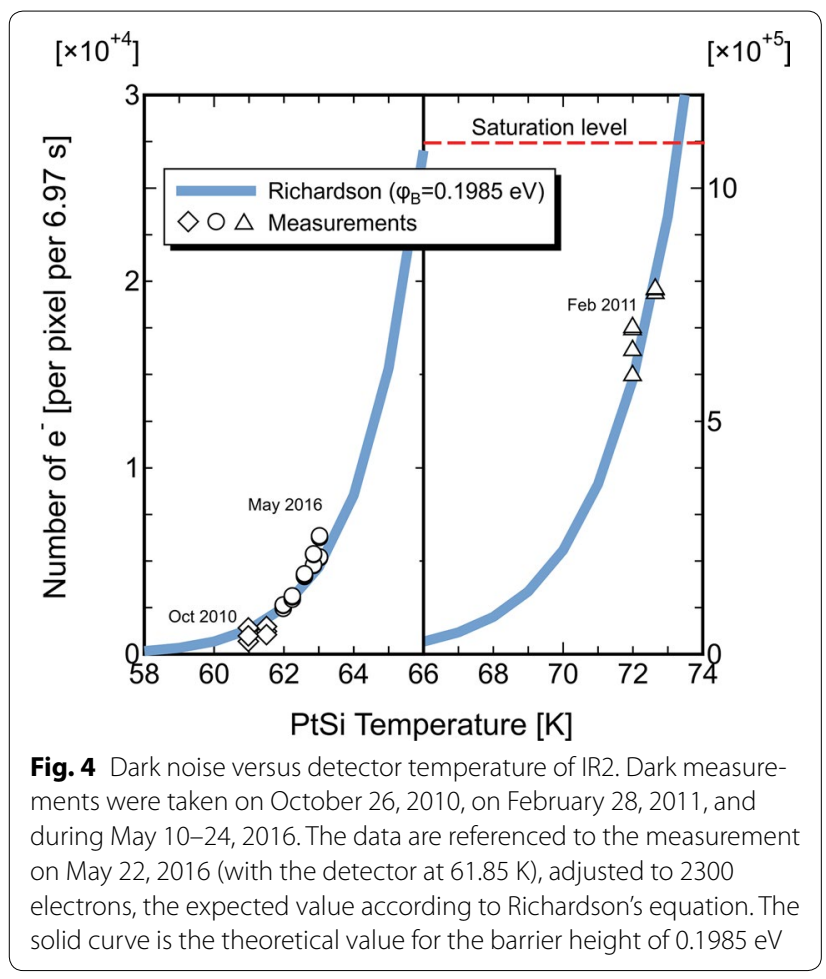

the other filter, $2.02 \mu \mathrm{m}$, the observation program 0x0A was executed only once by using an exposure time of 18.97 s. Standard aperture photometry was performed for individual images, and the results are summarized in Table 4.

In Paper I, the calibration coefficients were observationally estimated only for $1.65-\mu \mathrm{m}$ (the $\mathrm{H}$-band in highgain mode) and 2.02- $\mu \mathrm{m}$ filters, and the coefficients for the other filters $(1.735,2.26$, and $2.32 \mu \mathrm{m})$ were estimated by referring to design parameters such as the filter transmission curves, and quantum efficiency of the PtSi detector. The star fluxes in Table 4 were measured on images that were calibrated using such coefficients. The obtained fluxes are compared with fluxes from the 2MASS cata$\log$ (2MASS 2006). Since 2MASS only tabulates the H-band $(1.662 \mu \mathrm{m})$ and $\mathrm{Ks}$-band $(2.159 \mu \mathrm{m})$ values for the wavelength range of IR2, we linearly extended these two points to estimate the flux for the IR2 filters. Then, the measured (IR2) flux was divided by the estimated (2MASS) flux to obtain the IR2/2MASS ratio in Table 4. Interestingly, the ratios for all four filters fall within a narrow range, $0.60 \pm 0.06$. Antares is a red giant known to exhibit variability in brightness. Therefore, part of this discrepancy (non-unity of the IR2/2MASS ratio) may be attributed to the variable brightness of the star; however, the PSF issue, which is discussed in a later section, also exists. Actually, the H-band measurement of Antares (- 3.67 magnitudes on May 24, 2016) retrieved from the "AAVSO Light-Curve Generator" is 0.05 magnitude darker than that in 2MASS (Light-Curve Generator 1999). Using this brightness, instead of that of 2MASS, 
Table 4 Photometry of Antares

\begin{tabular}{|c|c|c|c|c|c|c|c|c|c|}
\hline \multirow[t]{2}{*}{ Filter $(\mu \mathrm{m})$} & \multicolumn{5}{|c|}{ IR2 (September 8, 2016) } & \multicolumn{3}{|c|}{ 2MASS catalog } & \multirow[t]{2}{*}{ IR2/2MASS ratio } \\
\hline & $N^{a}$ & $r^{a}$ & Meanc & $\sigma^{c}$ & Flux $^{d}$ & Magnitude & Flux $^{d}$ & Est. $^{\mathrm{d}}$ & \\
\hline 1.735 & 5 & 6 & 0.521 & 0.04 & 0.0205 & $-3.725(H)$ & 0.0352 & 0.0326 & 0.63 \\
\hline 2.26 & 5 & 4 & 0.228 & 0.03 & 0.0090 & $-4.100(\mathrm{Ks})$ & 0.0173 & 0.0137 & 0.66 \\
\hline 2.32 & 5 & 4 & 0.156 & 0.02 & 0.0062 & & & 0.0115 & 0.54 \\
\hline 2.02 & 1 & 4 & 0.338 & - & 0.0133 & & & 0.0223 & 0.60 \\
\hline
\end{tabular}

$\mathrm{H}$-band of 2MASS is $1.662(\mu \mathrm{m})$, and Ks-band is $2.159(\mu \mathrm{m})$

a Number of Antares images with the filter

a Radius of aperture used in photometry (pixels)

c Units are $\left(\mathrm{W} \mathrm{m}^{-2} \mathrm{sr}^{-1} \mu \mathrm{m}^{-1}\right)$

${ }^{d}$ Units are $\left(\mathrm{W} \mathrm{m}^{-2} \mathrm{~m}^{-1}\right)$

the above ratio 0.60 may be increased to 0.63 . It can be concluded that the internal consistency (of the order of $\pm 10 \%)$ of the calibration for all the IR2 filters has been confirmed observationally.

\section{Flat-field correction of images Overview}

The flat-field correction of IR2 images includes three steps. The first step is the correction for the "high-frequency" term of the flat-field response. The pre-launch flat data acquired in the laboratory are averaged and high-pass filtered such that only the "high-frequency" term is preserved. The obtained "high-frequency" flat, applied to images of Venus, is able to effectively correct for the pixel-to-pixel responses and to remove defects due to small particles on the detector (Paper I). The second step is the correction for the "low-frequency" flat-field response. The third step, which became necessary after we obtained many images of Venus in orbit, is intended to correct for brightness variations between quadrants of the detector. As explained in Paper I and in Akiyama et al. (1994), the IR2 detector is electrically divided into four quadrants in $2 \times 2$ format. Each quadrant is processed by a dedicated amplifier located on each corner, and the characteristics vary from one amplifier to another. The behavior seems to depend on the detector temperature, and this requires attention.

\section{"Low-frequency" flat field}

The "low-frequency" flat-field response was not well characterized before the launch. Because we had to place IR2 in a vacuum chamber, while on the ground, to perform all imaging tests, the small sapphire window did not allow the input of light to uniformly and fully illuminate the $12^{\circ} \times 12^{\circ} \mathrm{FOV}$ of the camera. (A "high-frequency" flat is easily obtained by subjecting the images to high-pass filtering.) Thus, we had to wait for an opportunity during which Venus (a bright light source) fills the IR2 FOV.
There were two such occasions (on October 8 and $19,2016)$ on which the spacecraft was very close to the dayside disk and IR2 was operated to capture $2.02-\mu \mathrm{m}$ images. The observation program 0x14 $(6.97 \mathrm{~s}$, single exposure) was used on October 8, and $0 \times 06$ (6.97 s, the median of three successive images) was used on October 19. Although the orbital motion of the spacecraft was expected to cause the image to become significantly smeared (making it as flat as possible), some inhomogeneous contrast markings continued to exist in the October 8 image (not shown). The October 19 image is, owing to median processing, much smoother (Fig. 5). We ensured that the brightness variation or dark ring in the image is not actually caused by the glory reported to occur in various wavelengths (García Muñoz et al. 2014; Petrova et al. 2015; Satoh et al. 2015), by examining the solar phase angles assigned to each pixel. The pattern we see in Fig. 5 cannot be attributed to glory because the solar phase angle at the image center is far from zero $\left(49^{\circ}\right)$; hence, point symmetry of the solar phase angles relative to the image center does not exist.

Therefore, we ascribe the brightness variation in the image (Fig. 5b) to the low-frequency term of the IR2 flatfield response. We approximate the brightness, $B(x, y)$, with a series of Legendre polynomials, $P_{i}(t)$, by assuming that the pattern is point symmetry relative to the image center $\left(x_{0}=512.5, y_{0}=512.5\right.$, and the maximum distance from the center is 723.4 pixels at the image corner):

$$
B(x, y)=\sum_{i=0}^{N} a_{i} P_{i}(t)
$$

where

$$
\begin{aligned}
& t=d / 725 \\
& d=\sqrt{\left(x-x_{0}\right)^{2}+\left(y-y_{0}\right)^{2}}
\end{aligned}
$$




\section{a Oct 19: full frame}

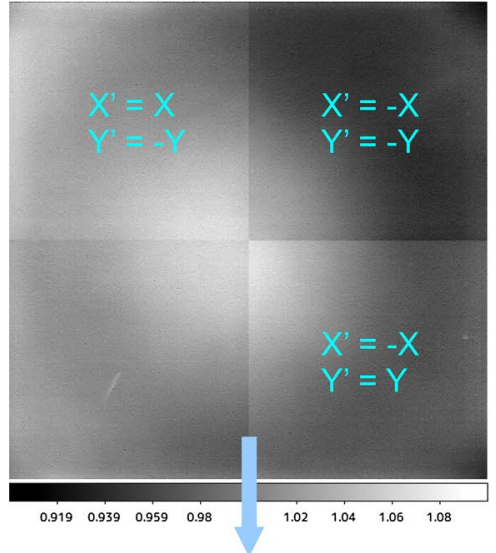

b Oct 19: Quadrants averaged
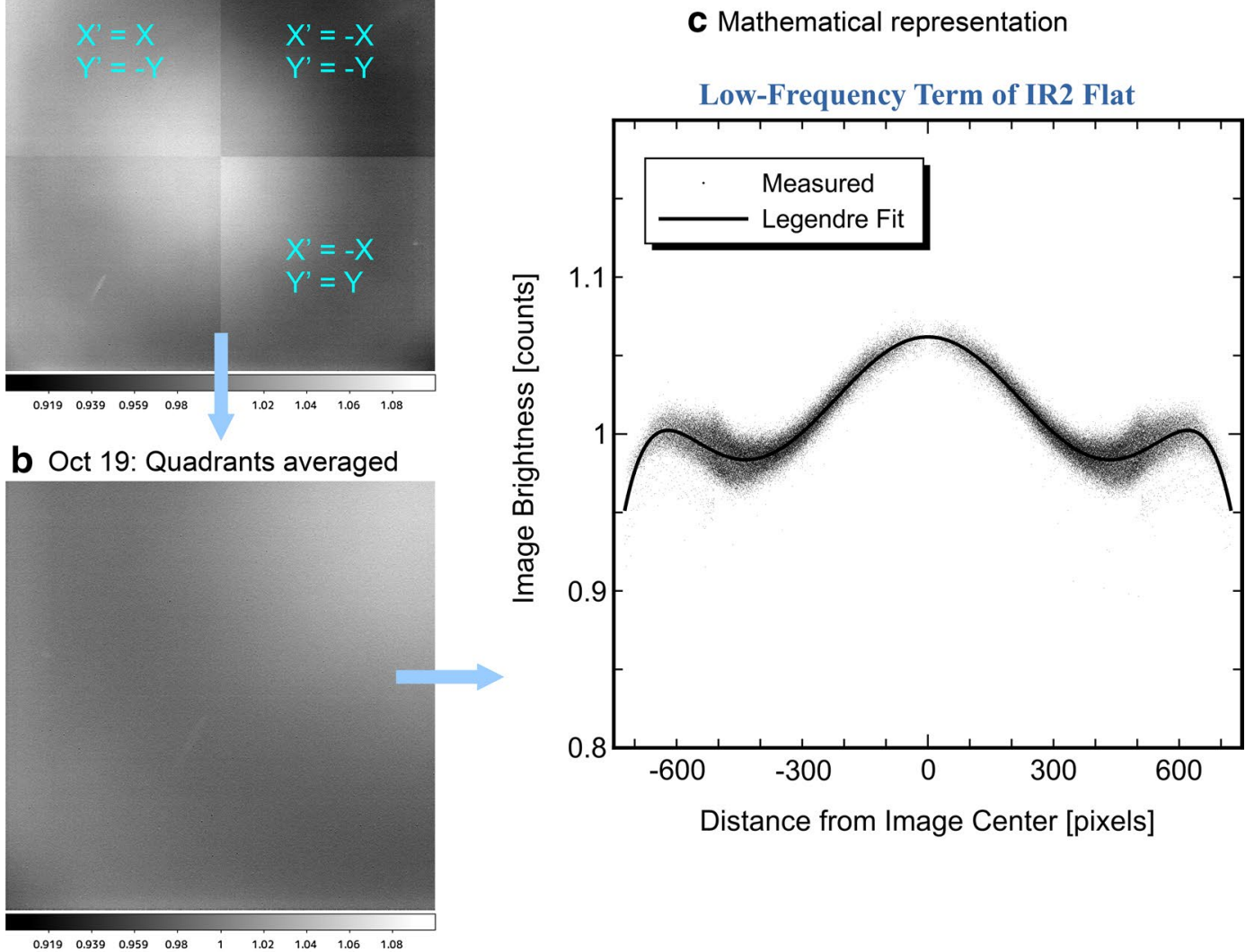

d Apr 25: before "low-freq" flat fielding e Apr 25: after "low-freq" flat fielding
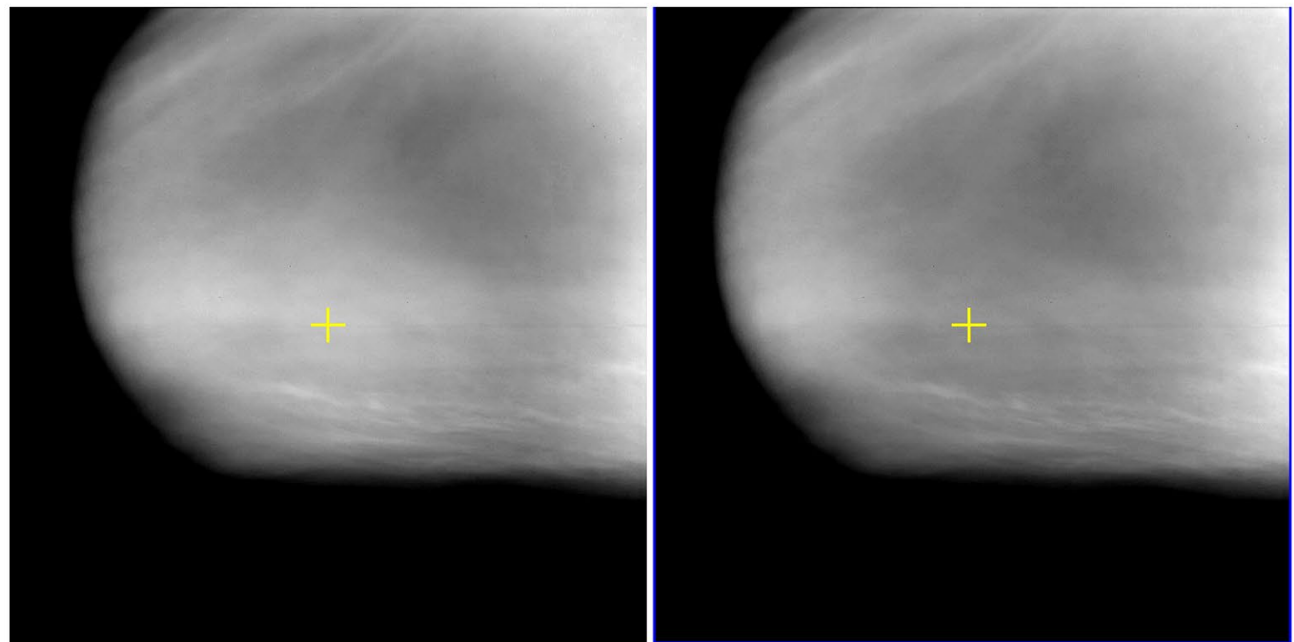

Fig. 5 Venus dayside disk image $(2.02 \mu \mathrm{m})$ taken from near the pericenter. a Original image acquired on October 19. b Four quadrants averaged to reduce the cloud pattern (considered as the "low-frequency" flat). c A mathematical representation of measured brightness variation as a function of distance from the image center. d Example of 2.02- $\mathrm{mm}$ image (acquired on April 25) with a large Venus disk. e After application of "low-frequency" flat. See text for details 
Figure $5 \mathrm{c}$ shows measured brightness (dots) and the mathematical representation (solid curve). Coefficients for the curve, $a_{i}$, are shown in Table 5 . Then, we have applied this low-frequency flat to a $2.02-\mu \mathrm{m}$ image in which Venus disk is sufficiently large to recognize such a pattern. In Fig. 5d, before the low-frequency flat is applied, Venus disk appears brighter around the image center with a hint of a surrounding dark ring. After the low-frequency flat is applied (Fig. 5e), the brightness of the Venus disk appears more symmetric to the sub-solar point (near the disk center) which can be understood as

Table 5 Coefficients for "low-frequency" flat

\begin{tabular}{lcll}
\hline Order $\boldsymbol{i}$ & Coefficient for $\boldsymbol{P}_{\boldsymbol{i}}(\boldsymbol{t})$ & Order $\boldsymbol{i}$ & Coefficient for $\boldsymbol{P}_{\boldsymbol{i}}(\boldsymbol{t})$ \\
\hline 0 & 1.009934 & 6 & -0.049436 \\
2 & -0.045019 & 8 & -0.002947 \\
4 & 0.038583 & & \\
\hline
\end{tabular}

larger penetration depths of sunlight near the sub-solar point.

We now conclude that the "low-frequency" term of the IR2 flat-field response has successfully been obtained.

\section{Temperature-dependent quadrant brightness}

The first IR2 image of Venus that spans multiple quadrants was obtained on March 14, $2016(2.02 \mu \mathrm{m})$. An examination of the pixel values near the boundary between quadrants, enabled us to determine the factors that cause quadrant boundaries to become almost invisible. However, applying the same factors to images obtained in subsequent months produced results that were quite unsatisfactory and additional correction factors, varying from image to image, were definitely required. Plotting these factors against the detector temperature led to a surprising result (Fig. 6).

It is obvious that the correction factors depend on the detector temperature. By approximating these curves
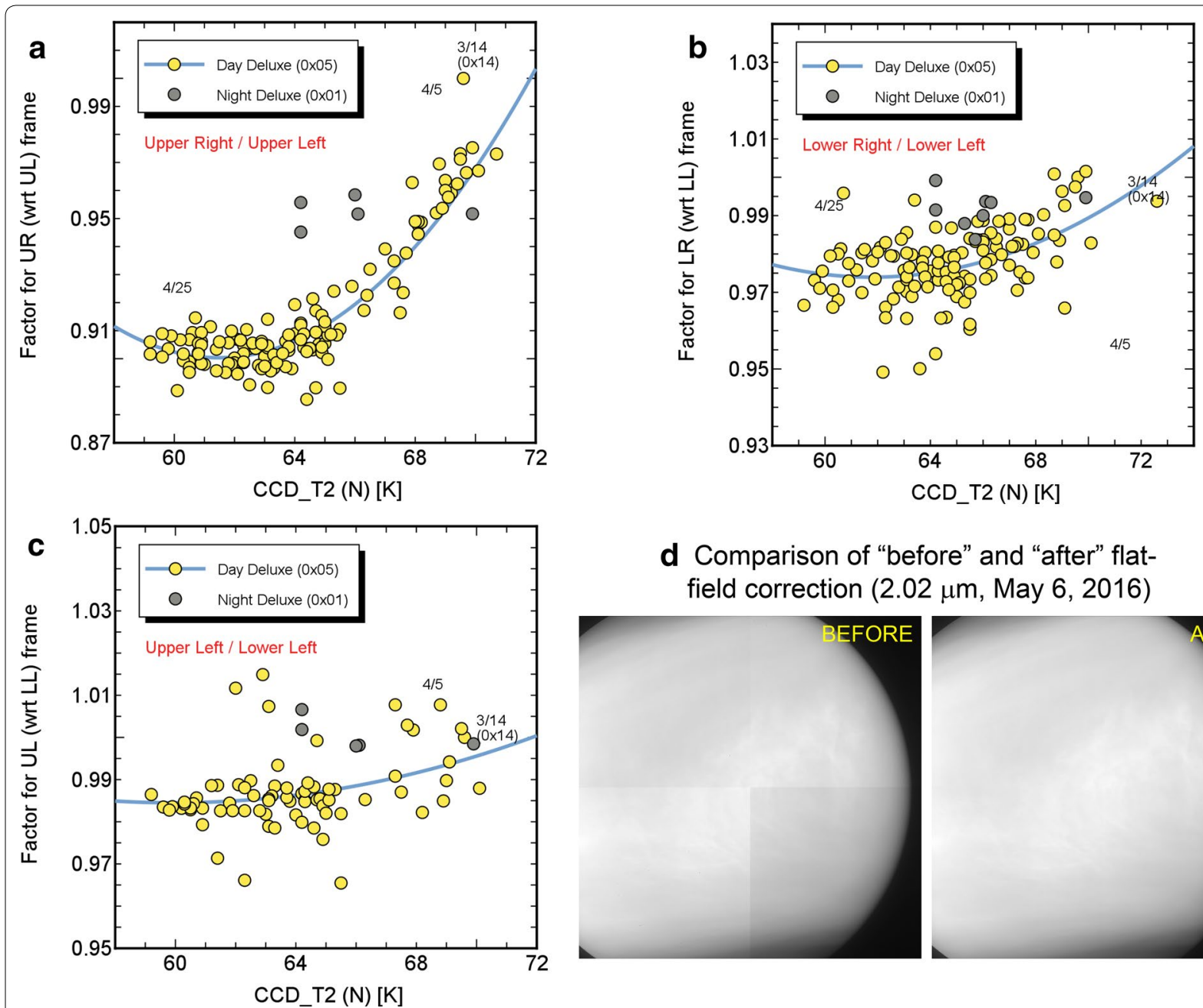

d Comparison of "before" and "after" flatfield correction (2.02 $\mu \mathrm{m}$, May 6, 2016)
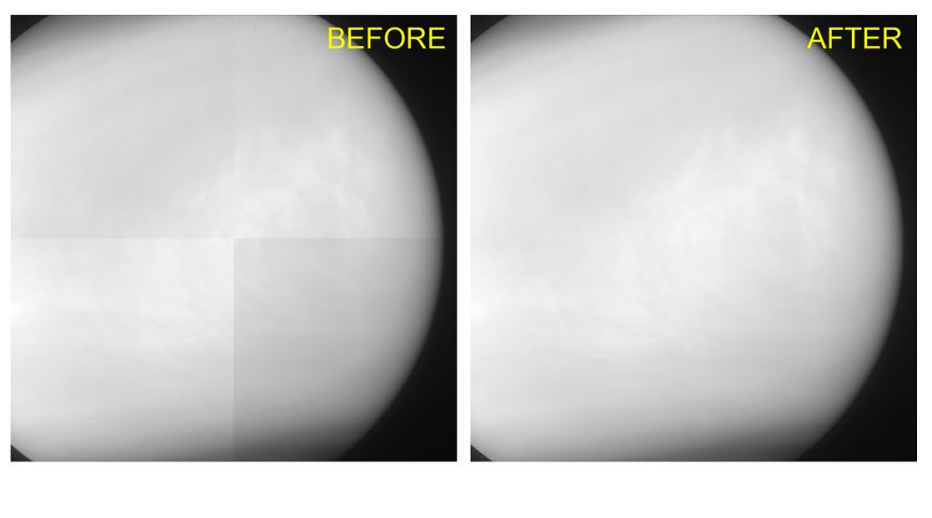

Fig. 6 Temperature dependence of correction factors for brightness variation between quadrants. a Correction between upper-right and upperleft quadrants. b Correction between lower-right and lower-left quadrants. c Correction between upper-left and lower-left quadrants. $\mathbf{d}$ A demonstration of the brightness correction between four quadrants 
with quadratic functions (version 0.5, dated September $20,2016)$, we corrected the brightness variation between quadrants by implementing the following three steps:

(1) The brightness variation between the upper two quadrants (Fig. 6a) is adjusted with the following equation:

$$
f_{1}=\sqrt{ }\left(4.4164-1.1439 \times 10^{-1} T+9.3038 \times 10^{-4} T^{2}\right) .
$$

Then, the upper-left quadrant is divided by $f_{1}$, whereas the upper-right quadrant is multiplied by $f_{1}$.

(2) The brightness variation between the lower two quadrants (Fig. 6b) is adjusted with the following equation:

$$
f_{2}=\sqrt{ }\left(1.8467-2.8255 \times 10^{-2} T+2.2869 \times 10^{-4} T^{2}\right) .
$$

Then, the lower-left quadrant is divided by $f_{2}$, whereas the lower-right quadrant is multiplied by $f_{2}$.

(3) The brightness variation between the upper and lower quadrants (Fig. 6c) is adjusted with the following equation:

$$
f_{3}=\sqrt{ }\left(1.3774-1.3110 \times 10^{-2}+1.0936 \times 10^{-4} T^{2}\right) .
$$

Then, the lower quadrants are divided by $f_{3}$, whereas the upper quadrants are multiplied by $f_{3}$.

After this operation, the brightness over all quadrants becomes almost uniform (Fig. 6d).

\section{Known issues (electrical artifacts)}

The readout procedure of the IR2 detector entails switching the four quadrants in sequence: after reading a pixel from the lower-left (LL) quadrant, followed by a pixel from the lower-right (LR) quadrant at a similar position, and the process is repeated for the upper-left (UL) and upper-right (UR) quadrants. This LL-LR-UL-UR cycle is repeated until all pixels are read out. As a result, when an extremely saturated pixel is read from a quadrant, subsequent pixels (even from unsaturated quadrants) are affected, causing noticeable artifacts that are axisymmetric to the horizontal and vertical centers. This problem is more serious for the night-side data in which very intense light from the illuminated Venus disk saturates pixels, thereby yielding the electrical artifacts. The artifacts become further noticeable after contamination by light originating from the intense dayside disk is removed (see next section).

\section{Point-spread function issue}

\section{Overview}

Soon after we started imaging the night-side of Venus with IR2, a significant amount of light was found to be spreading from the dayside disk, contaminating pixels far from the source. An examination of these images of Venus as well as pre-launch laboratory images led us to the conclusion that this peculiar point-spread function (PSF) is caused by multiple reflections of light inside the detector substrate. Only a fraction of incoming light to the PtSi detector yields photoelectrons at the PtSi layer of a few nanometer thick, leaving a much larger fraction of light unused (Akiyama et al. 1994). This explains why the quantum efficiency of this sensor is not high. Consequently, multiple reflections of "unused" light can occur in the Si substrate (transparent for 2- $\mu \mathrm{m}$ NIR light), resulting in the peculiar PSF seen in the IR2 images.

\section{Halation around the point source: evidence of total reflection}

When multiple reflections in the PtSi detector were suspected, we re-examined the pre-launch laboratory images and found a good example of the phenomenon as shown in Fig. 7a. The figure shows an $\mathrm{H}$-band $(1.65-\mu \mathrm{m})$ image of a view of the night scenery from the laboratory. (The port of the vacuum chamber with the sapphire window intentionally faced a window of the laboratory to enable such imaging.) Halation rings around the bright city lights are apparent, evidence that total reflection does occur in the detector. The inner radius of these halation rings ( 15 pixels), together with the pixel size $(17 \mu \mathrm{m})$ and the refraction index of $\mathrm{Si}(3.46$ at $1.6 \mu \mathrm{m})$, is completely consistent with the $400 \mu \mathrm{m}$ thickness of the Si substrate of the IR2 detector.

The cross-pattern extending both horizontally and vertically suggests that pixels aligned in rows and columns act as guiding channels for the reflected light.

\section{Approximation of IR2 PSF with a mathematical function}

Considering the aforementioned evidence, we constructed a mathematical approximation of the PSF of IR2 with the point-symmetric term and the axisymmetric terms (in the $X$ - and $Y$-directions). The mathematical function is as follows:

$$
a_{\text {total }}=g_{1} a_{r}(r)+g_{2} a_{X}(X) \times a_{Y}(Y)
$$

where

$$
\begin{aligned}
& r^{2}=X^{2}+Y^{2} \\
& X=x-x_{0} \\
& Y=y-y_{0}
\end{aligned}
$$

and $\left(x_{0}, y_{0}\right)$ is the center of the PSF.

The point-symmetric term, $a_{r}(r)$, is in the following form:

$$
a_{r}(r)=\frac{1}{\pi} \frac{\Gamma_{1}}{2} \frac{1}{\left[r^{2}+\left(\Gamma_{1} / 2\right)^{2}\right]^{e_{1}}}
$$



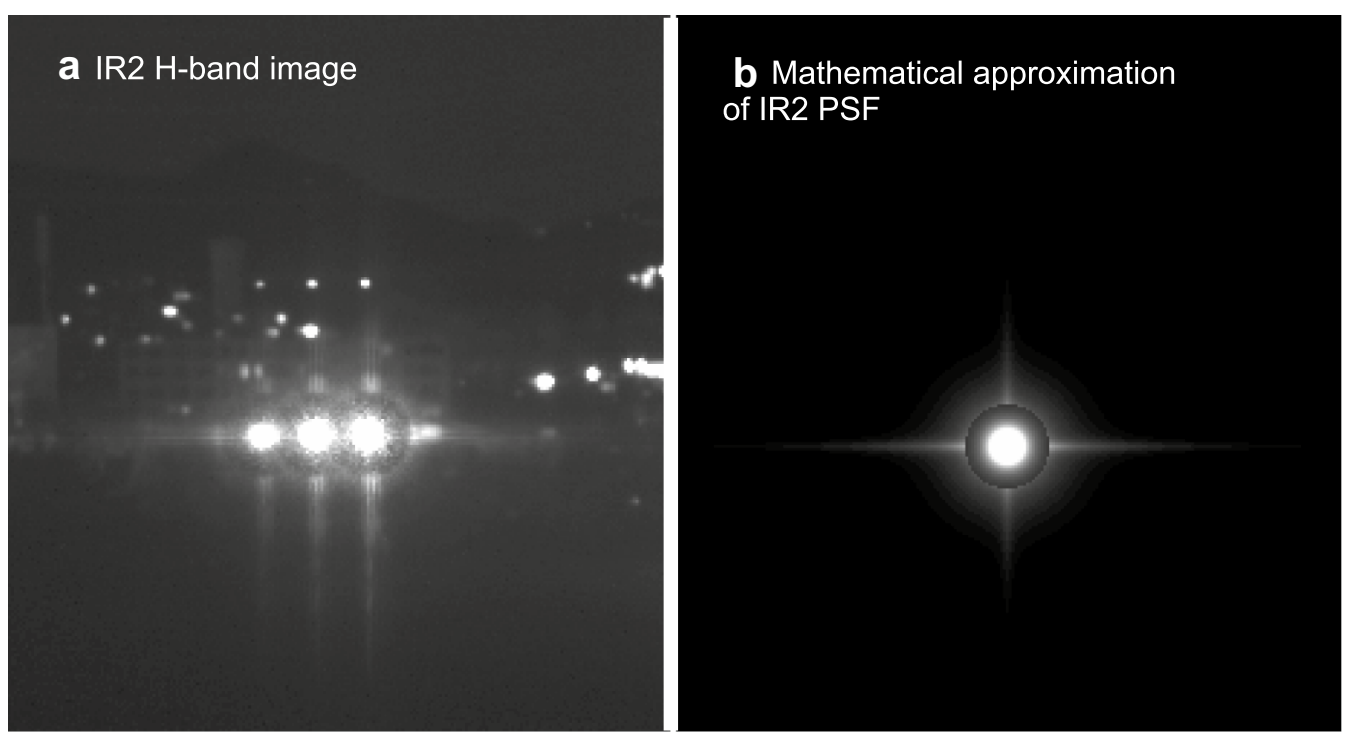

Fig. 7 Point-spread function (PSF) of IR2. a Pre-launch H-band image, taken in the evening through the window of the laboratory, exhibiting halation rings around the bright city lights (three in row). b Mathematical approximation of the IR2 PSF as described in the text. This is intended to be similar to the pattern seen in the real image

where $\Gamma_{1}$ and $e_{1}$ are tunable parameters. Note that this function is the so-called Moffat function (Howell 2000) and reduces to a Lorentzian when $e_{1}=1$.

The axisymmetric terms, $a_{X}(X)$ and $a_{Y}(Y)$, are expressed similarly:

$$
a_{X}(X)=\frac{1}{\pi} \frac{\Gamma_{2}}{2} \frac{1}{\left[X^{2}+\left(\Gamma_{2} / 2\right)^{2}\right]^{e_{2}}}
$$

and

$$
a_{Y}(Y)=\frac{1}{\pi} \frac{\Gamma_{2}}{2} \frac{1}{\left[Y^{2}+\left(\Gamma_{2} / 2\right)^{2}\right]^{\left(e_{2} c\right)}}
$$

where $\Gamma_{2}$ and $e_{2}$ are common parameters to $a_{X}(X)$ and $a_{Y}(Y)$ with $c$ only appearing in $a_{Y}(Y)$. This additional parameter allows the tail shape of the PSF in $Y$ to differ slightly from that in $X$, intended to partially incorporate the structural difference between rows and columns in the real detector.

The region within the halation ring (a radius of 15 pixels) is expressed as follows:

$$
a_{\text {total }}=g_{1} a_{r}(r) \times f
$$

Note that real images do not exhibit the cross-pattern in this region; thus, it is not included in the function. Additionally, a factor $f(<1)$ is applied to the point-symmetric term to describe the loss of singly reflected light from the surface of the Si substrate (Fig. 7a).

\section{Performance of constructed PSF}

A tentatively determined PSF is displayed in Fig. 7b with the same magnification scale as that in Fig. 7a. Parameter values are summarized in Table 6 . The first test is to remove contamination of light spreading from the intense dayside disk from the night-side image (2.26 $\mu \mathrm{m}$ acquired on March 28, 2016). A synthetic dayside disk image is generated by assuming Lambertian intensity distribution and then convolved with the PSF of Fig. 7b. The convolved image is then subtracted from the original image. A "before and after" comparison is demonstrated in Fig. 8a, b. The contrast in the nightside disk is improved, and future photometric studies with IR2 data should incorporate similar restoration procedures. Note that the artifacts mentioned in 5.5 become obvious with axisymmetry to the horizontal and vertical centers.

Although restoration of the night-side image primarily validates the extended tail region of the PSF, deconvolution of the dayside image tests both the sharp core and the tail of the PSF. Figure 8c, d demonstrates the extent

Table 6 Parameters to describe PSF

\begin{tabular}{llllll}
\hline$g_{1}$ & 9.0 & $g_{2}$ & 1.0 & $c$ & 1.2 \\
$\Gamma_{1}$ & 0.80 & $\Gamma_{2}$ & 3.0 & $f$ & 0.60 \\
$e_{1}$ & 1.25 & $e_{2}$ & 0.70 & & \\
\hline
\end{tabular}



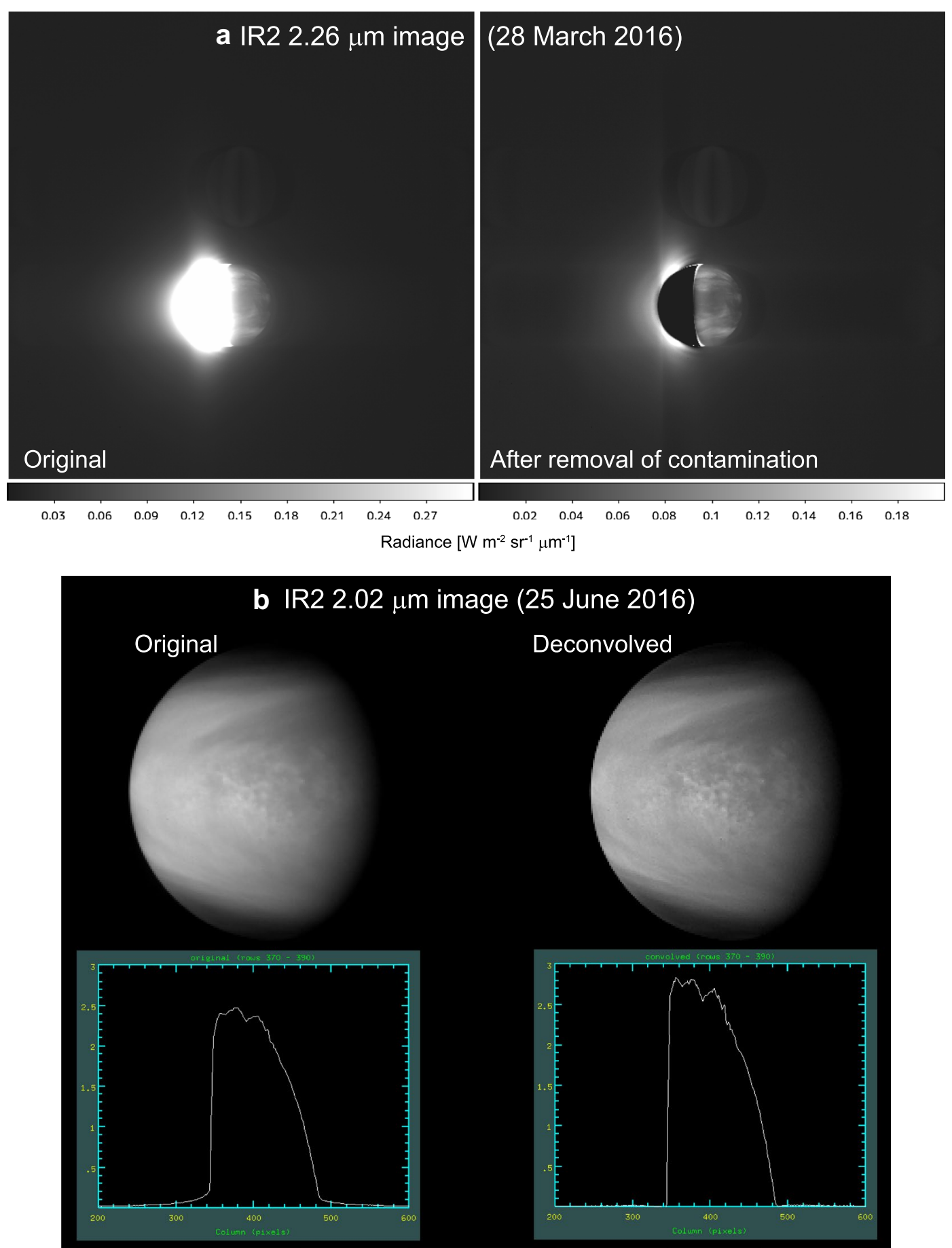

Fig. 8 Application of IR2 PSF to Venus images. a Demonstration of removal of contamination of the night-side disk by the intense dayside disk $(2.26 \mu \mathrm{m}$, acquired on March 28, 2016). The procedure is described in the text. After removal of the contamination, the electrical artifacts of the IR2 image become more noticeable. b Demonstration of deconvolution (Lucy-Richardson algorithm) performed on a 2.02- $\mu$ m dayside disk (June 25, 2016). The PSF in Fig. 7b is used for this restoration, and no significant artifact is noticed

to which a $2.02-\mu \mathrm{m}$ dayside image is improved both in terms of the contrast (resolution) of the cloud features on the disk and in terms of recollecting the light spread over the pixels of deep space. Therefore, this restoration should be useful for morphological studies, cloud-top altimetry, and photometry with the $2.02-\mu \mathrm{m}$ data. 


\section{Implication to absolute calibration}

Once the shape of the PSF is inferred (even though tentatively), it is possible to estimate how much light from the source actually falls within the aperture used in photometry. We have found that $\sim 64 \%$ of light falls in the aperture with a 4-pixel radius and it increases to $\sim 68 \%$ when the aperture radius is 6 pixels. These numbers almost coincide with the IR2/2MASS ratios in Table 4. Therefore, those ratios may include both the variability of the star brightness and the measurement efficiency (significant for point-source objects but less significant for extended objects) owing to the extended shape of the PSF. The conclusion about the internal consistency of absolute calibration nonetheless remains unchanged.

Please note that the parameter values in Table 6 as well as the form of function used to describe the PSF is not final as these have been tested on at limited amount of data. Therefore, there is no guarantee that these values are appropriate for all data. We provide this information in the hope that it serves as a useful starting point for researchers who are to analyze the IR2 data.

\section{Geometrical characteristics}

\section{Astrometry of star-field images}

Paper I reports a set of astrometric results with position measurements for five bright stars in one star-field image. In the work reported in this paper, we performed more comprehensive astrometry for three star-field images in the H-band $(1.65 \mu \mathrm{m})$, each of which contains more than 30 star position measurements. The measured positions are compared with those in the 2MASS star catalog. The results are summarized in Table 7 .

It is noteworthy that the results obtained for the three images are quite consistent with each other. The scale factor 0.951 (an average of three) is relative to the nominal scale of $0.012^{\circ}$ per pixel (before binning). Therefore, the measured scale, which is $0.0114^{\circ}$ per pixel, translates to a focal length of $85.35 \mathrm{~mm}$ and is consistent with the result of five-star astrometry $(85.42 \mathrm{~mm})$ in Paper I. Because the number of stars in one image is much larger than in the report in Paper I, we are now able to plot residual vectors to examine whether residuals behave systematically (an indication of distortion). Figure 9 is one example in which the residual vectors are magnified by a factor of 40 . This plot, together with the very small RMS residuals in Table 7, suggests that the geometrical distortion of an IR2 image is negligible, thereby confirming the conclusion reached in Paper I but with greater confidence.

\section{Updated focal length with wavelength dependency}

The triplet lens of IR2 has remaining chromatic aberration; thus. the focal length varies from one filter to another. The theoretical focal lengths provided by the manufacturer (Nikon) for four filters used for imaging Venus $(1.735,2.02,2.26$, and $2.32 \mu \mathrm{m})$ at two temperatures (293 and $170 \mathrm{~K}$ ) are shown in Fig. 10. Because the design parameters for the optics are not disclosed, we approximated these discrete points with the quadratic functions of the wavelength by using one function for each temperature. The focal lengths for filters other than $1.65 \mu \mathrm{m}$ were estimated by applying factors from the $170-\mathrm{K}$ curve to the astrometric value, $85.35 \mathrm{~mm}$ (at $1.65 \mu \mathrm{m})$, and are summarized in Table 8.

\section{Image navigation refinement}

A number of factors, such as thermal distortion of the spacecraft and the instrument, cause the image navigation data to deviate from the ideal values. Therefore, correction becomes necessary to enable accurate

Table 7 Astrometry of star-field images

\begin{tabular}{|c|c|c|c|c|c|c|}
\hline \multicolumn{2}{|c|}{ Images (Oct. 23, 2010) } & \multicolumn{2}{|c|}{ Center coordinate } & \multirow[t]{2}{*}{ Scale (\% of nominal) } & \multirow[t]{2}{*}{ Tilt $\left(^{\circ}\right)$} & \multirow[t]{2}{*}{ RMS (pixels) } \\
\hline & Time (UT) & $\mathrm{RA}\left({ }^{\circ}\right)$ & $\operatorname{DEC}\left({ }^{\circ}\right)$ & & & \\
\hline \multirow[t]{3}{*}{$1(33)^{\mathrm{a}}$} & 00:37:05 & 115.788 (Obs) & 24.0930 (Obs) & 0.952 & -0.702 & 0.88 \\
\hline & $00: 39: 17$ & 116.122 (SPICE) & 24.1096 (SPICE) & & & \\
\hline & $00: 41: 29$ & & & & & \\
\hline \multirow[t]{3}{*}{$2(35)^{\mathrm{a}}$} & $01: 27: 26$ & 127.458 (Obs) & 21.8535 (Obs) & 0.950 & -0.709 & 0.88 \\
\hline & 01:29:38 & 127.798 (SPICE) & 21.8142 (SPICE) & & & \\
\hline & $01: 34: 15$ & & & & & \\
\hline \multirow[t]{3}{*}{$3(38)^{a}$} & 00:11:55 & 109.836 (Obs) & 24.8835 (Obs) & 0.951 & -0.660 & 0.91 \\
\hline & $00: 14: 07$ & 120.168 (SPICE) & 24.9046 (SPICE) & & & \\
\hline & $00: 16: 19$ & & & & & \\
\hline Average result & & $X:-0.304\left(^{\circ}\right)$ & $Y:+0.052\left(^{\circ}\right)$ & 0.951 & $-0.690\left(^{\circ}\right)$ & \\
\hline
\end{tabular}

a Numbers in parentheses are number of star position measurements in the image 


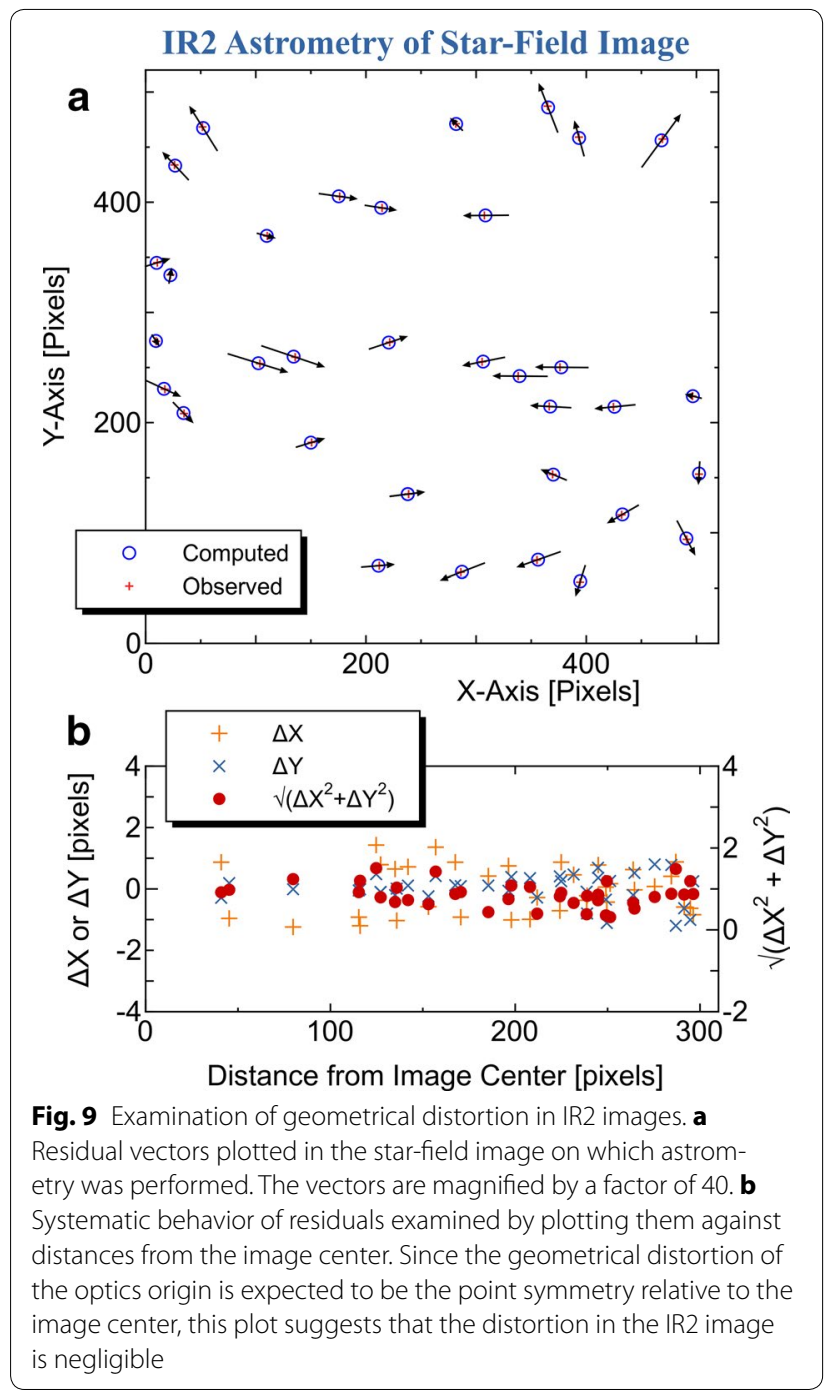

geographical mapping of the image data. In the data products from the Akatsuki project, "Level-2" does not incorporate this correction, whereas "Level-3" does. The correction in the Level-3 product is achieved by fitting the planetary disk limb to each image, as described by Ogohara (2017). The limb fitting is found to be quite successful for the dayside images (manuscript in preparation). However, it continues to remain challenging for the IR2 night-side data, because the night-side limb is often obscure and demands careful parameter tuning. The limb fitting is available only when the image captures a sufficient fraction the planetary limb. Therefore, the users of the IR2 data products are advised to be aware of the possible large navigation error when the fitting is unavailable, which is found by a flag.

The result of the Level-3 dayside limb fitting indicated that the typical amount of correction necessary is a few pixels, which corresponds to a deviation of the pointing

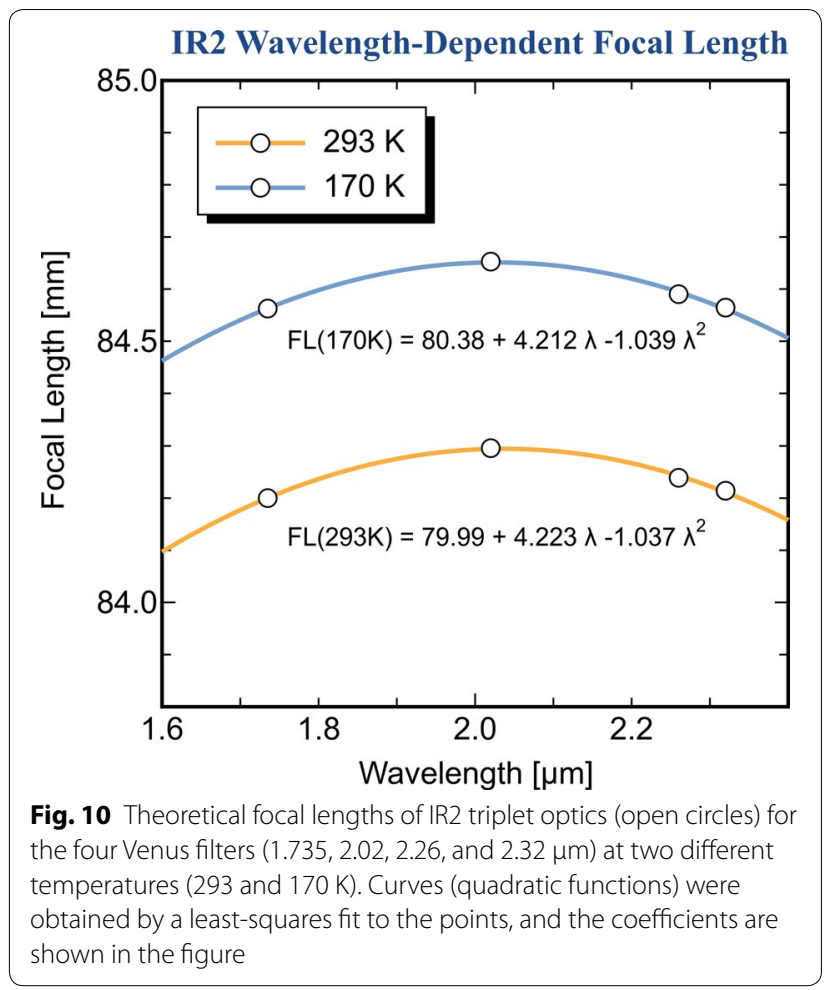

Table 8 Updated focal lengths

\begin{tabular}{lll}
\hline Filter $(\boldsymbol{\mu m})$ & Factor & Focal length $(\mathbf{m m})$ \\
\hline 1.65 & 1.0 (reference) & 85.35 (astrometry) \\
1.735 & $+0.07 \%$ & 85.41 \\
2.02 & $+0.17 \%$ & 85.50 \\
2.26 & $+0.11 \%$ & 85.44 \\
2.32 & $+0.07 \%$ & 85.41 \\
\hline
\end{tabular}

direction to the order of $10^{-3} \mathrm{rad}$. The dayside limb fitting also indicated that the overall effect of thermal distortion can be understood to be a function of the local time of the sub-spacecraft longitude, which roughly corresponds to the spacecraft attitude with respect to the sunlight vector.

Given the uncertainty in the night-side limb fitting, we devised an alternative approach to correct the pointing direction as described in what follows, and the results are used in the initial studies with the IR2 night-side images (e.g., Horinouchi et al. 2017a). First, the local time dependence mentioned above was applied to correct the pointing direction. This step is not mandatory, but it helps narrowing the search ranges in the next step. Second (and last), the mean radial gradient along the limb was optimized to correct the pointing direction as follows. The image navigation data include the 
sub-spacecraft point in the image coordinate; thus, we varied it in two dimensions; the particular parameters we chose range from -7 to 7 and from -5 to 5 pixels along the $x$ - (predominantly east-west) and $y$ (predominantly north-south)-coordinates of the image, respectively, in intervals of 0.25 pixels. For each of the specified sub-spacecraft points, we computed the inward radial gradient of radiance and averaged it along the limb derived from the specification, and we chose the correction to maximize the mean value. When doing so, the radius of the "limb," which is the point at which the radial radiance gradient is maximized, was empirically assumed to be $6126 \mathrm{~km}$ (from an inspection with a 6-km interval), and the night-side limb within latitudes in the range $\pm 75^{\circ}$ was used; further, a negative gradient (i.e., for which the radiance increases outward) and outliers exceeding three standard deviations were excluded for the purpose of averaging.

Subjective visual inspection of the results indicated that the correction accuracy exceeds 0.5 pixels. As an objective evaluation, we conducted cloud-tracking by using images acquired at 2-h intervals by employing the methods of Ikegawa and Horinouchi (2016) and Horinouchi et al. (2017b), and we computed the unbiased standard deviation of the resultant mean winds within each of Earth's calendar days in July to August, 2016; for example, we used five image pairs from the six images taken at 9, $11, \ldots, 19-\mathrm{h}$ UTC on a given day. The resultant standard deviations are $1-4 \mathrm{~m} / \mathrm{s}$, which correspond to distances of $10-30 \mathrm{~km}$. Interestingly, we did not find a clear dependency on the apparent diameter of Venus in the images, which would indicate that the correction accuracy tends to be higher when the apparent diameter is smaller; the above-mentioned standard deviation values correspond to $0.1-0.3$ pixels when the diameter of Venus is 120 pixels, for example. This insensitivity is partly attributed to the standard deviation including a contribution from factors other than the pointing error. However, a close inspection of some night-side image sequences when the diameter of Venus ranges from 400 to 500 pixels suggested that the pointing error can sometimes approximate 1 pixel.

\section{Quality of the data}

\section{Performance on fine-scale cloud features}

A set of example images are displayed in Fig. 11. The sub-frame of a $2.26-\mu \mathrm{m}$ night-side image (acquired on March 25, 2016) in Fig. 11a exhibits complex atmospheric dynamics in the middle latitude of the northern hemisphere. The bright spot near the upper-left corner ( $36^{\circ} \mathrm{N}$ latitude) emits radiance of $\sim 0.5 \mathrm{~W} \mathrm{~m}^{-2} \mathrm{sr}^{-1} \mu \mathrm{m}^{-1}$, suggesting that the optical thickness of the cloud approximates 18. (A continuum absorption coefficient of
$2.5 \times 10^{-8} \mathrm{~cm}^{-1}$ amagat $^{-2}$ is assumed.) This may likely be a region of strong downwelling motion.

Another 2.26- $\mu \mathrm{m}$ night-side image (acquired on April 15, 2016, also a sub-frame) in Fig. 11b is unusual with a discontinuous change in cloud opacity and a very finescale structure at the contrast boundary. An intensity profile for a portion of the image (indicated with a circle) is plotted in which 3 dark ribbons are identified. These ribbons appear with a cycle of only 3 pixels, a good demonstration of performance of the IR2 optics. Assuming the modulation-transfer function of 0.5 (the optics plus the detector), the intensity slope in the background of these ribbons may be reconstructed (solid circles in yellow). Such information may be used in the future quantitative analysis to investigate what these ribbons are as well as the discontinuous change in cloud opacity.

An example of a 2.02- $\mu \mathrm{m}$ dayside image (acquired on May 17, 2016) is shown in Fig. 11c together with its deconvolved version in Fig. 11d. The image contains a meridionally extended feature (tilted westward in the north) that has been identified as being originated from Beta Regio, a phenomenon similar to that as reported with LIR observations (Fukuhara et al. 2017). In addition to the main feature, fainter but repeating features also exist in parallel to the main feature. These may be generated by gravity waves propagating from the deeper atmosphere (manuscript in preparation).

The detailed physical interpretation of these phenomena will be discussed in separate papers.

\section{Photometric quality \\ Night-side observations}

As mentioned in "Point-spread function issue" section, the night-side data are affected by the light from the intense dayside disk. One approach to lessen this problem is to subtract, from the night-side data, the estimated contamination as demonstrated in "Performance of constructed PSF" section. Another approach is to suppress the light at the time of image acquisition by offsetting the dayside disk such that it is out of the range of the detector, which involves precise control of the spacecraft attitude. We refer to the latter approach as "night-side optimized" observation.

Figure 12 compares both approaches on $1.735-\mu \mathrm{m}$ data (acquired on July 22, 2016) in a scatter plot. The correlation coefficient is good $(r=0.89)$ although both types of data seem to have an extent of bias above true zero. This may be removed by examining the brightness of deep space pixels immediately outside the night-side disk of Venus. We conclude that consistent results can be obtained using both approaches for photometrical 


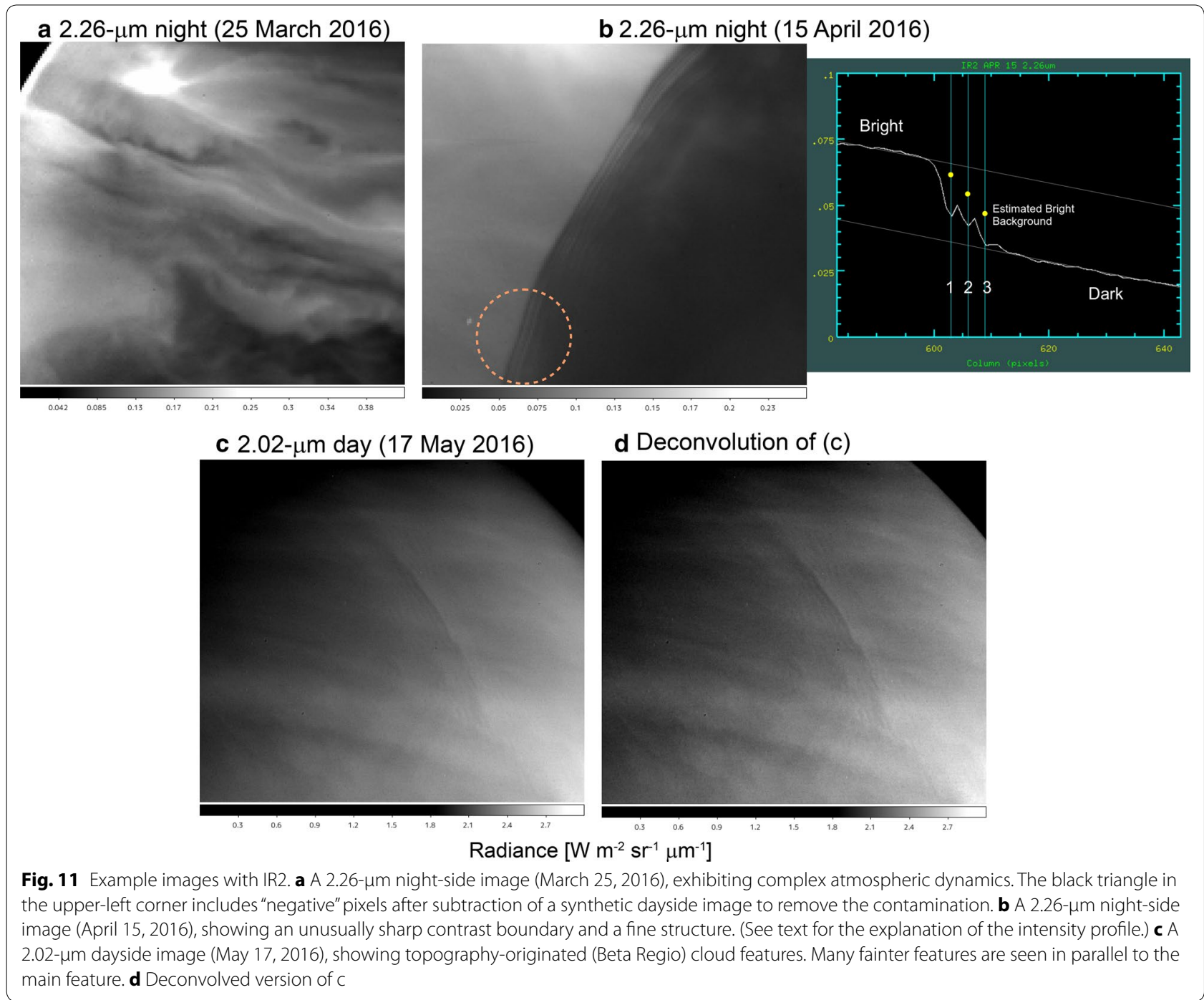

studies on the night-side disk. Note that the maximum absolute value in Fig. 11, $0.3 \mathrm{~W} \mathrm{~m} \mathrm{sr}^{-1} \mu \mathrm{m}^{-1}$, is consistent with previous Galileo/NIMS measurements (Grinspoon et al. 1993). This also is supportive of the validity of absolute calibration of IR2 data. (Extended objects are expected to be less affected by the shape of PSF.)

\section{Dayside observations}

The photometric stability of dayside observations is evaluated by performing disk-integrated flux measurements for the period May 21-25, 2016. A set of two images, separated by $4 \mathrm{~h}$, were acquired on each day. The images are deconvolved with the PSF described above in order to recover the loss of flux to the deep space pixels. The result is shown in Fig. 13. Day-by-day variations are obvious as the solar phase angle $(\alpha)$ changes from $\sim 21^{\circ}$ (May
21) to $\sim 3^{\circ}$ (May 25), with the brightness ratio between $21^{\circ}$ and $3^{\circ}$ phase angles consistent with the Akatsuki/IR2 measurements of 2011 (Satoh et al. 2015). The consistency between two measurements in a single day is superb because the two points are indistinguishable, for both May 21 and 22, in the plot. Therefore, it is concluded that high-precision photometry of the dayside Venus disk is possible with IR2.

\section{Conclusion}

The quality of data acquired during the first year by the IR2 camera, which is attached to a spacecraft orbiting Venus, together with a performance evaluation of the instrument, is reported in greater detail than in Paper I. This work enabled us to learn to stabilize the operation of the cryo-cooler of IR2 while achieving the desired cooling performance. Both dayside $(2.02 \mu \mathrm{m})$ and night-side 


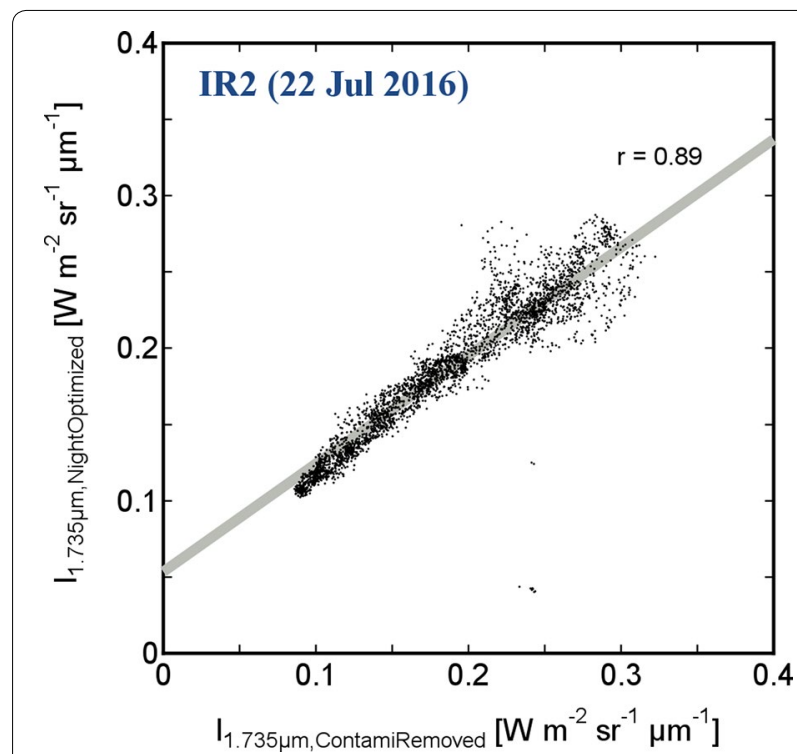

Fig. 12 Scatter plot comparing an image acquired at $1.735 \mu \mathrm{m}$ after removal of contamination and an optimized night-side image (separated by $1.5 \mathrm{~h}$ on July 22,2016$)$. The correlation coefficient is good $(r=0.89)$ with both types of data biased above true zero

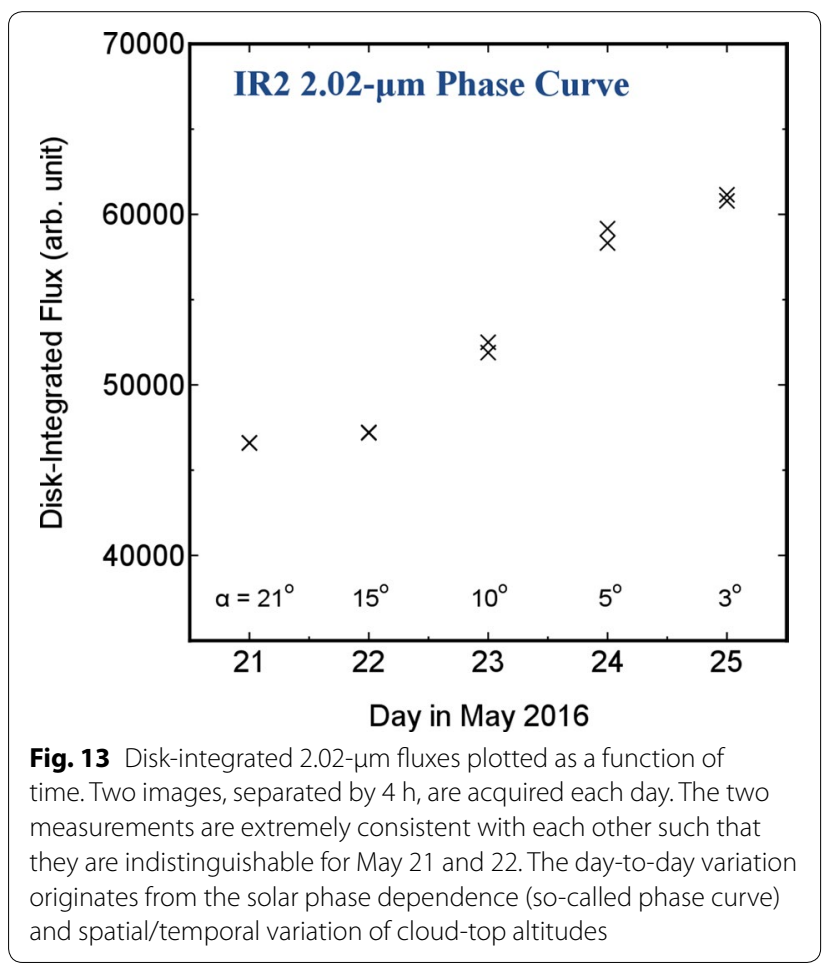

$(1.735,2.26$, and $2.32 \mu \mathrm{m})$ images of Venus were obtained. The scientific usefulness of the data was increased by validating the absolute calibration and by modeling the point-spread function (PSF). The procedure to correct for the brightness variation between the detector quadrants (temperature dependent) was also established. Many of these issues were previously not well known because of limitations of both the "pre-launch" test environment and the spacecraft attitude (to survive the severe thermal conditions) while waiting another five additional years until the VOI-R.

Now, we are able to declare with confidence that the production of IR2 data has been stabilized and the quality of data is assured for subsequent scientific studies.

\begin{abstract}
Abbreviations
PtSi: platinum silicide; PSF: point-spread function; VEx: Venus Express; VIRTIS: Visible, Infrared and Thermal Imaging Spectrometer; VOI-R: Venus orbit insertion-revenge; AD: analog-digital; FET: field-effect transistor; 2MASS: Two Micron All Sky Survey; AAVSO: American Association of Variable Star Observers; FOV: field of view; RMS: root-mean-square; NIMS: Near-Infrared Mapping Spectrometer
\end{abstract}

\section{Authors' contributions}

TS, as the Principal Investigator of IR2, was responsible for all elements of this manuscript and the underlying work. TMS, MN, YK, MU, MS, GLH, and TN, as the IR2 team members, enabled IR2 observations and contributed to the characterization of the instrument. YS performed the photometry of the star images. KT and KeS conducted the parameter search for PSF. TE developed the level-2 data conversion program for IR2. TH refined the image navigation by limb-fitting method. TI, AY, NI, TA, CH, TF, KO, HA, KoS, HK, and SO enabled orbit insertion of the spacecraft and subsequent scientific operations. NH, MY, SM, and $Y Y$ devoted their time to the data-processing pipeline and archiving. All authors read and approved the final manuscript.

\section{Authors' information}

TS is the Principal Investigator of IR2 and at the same time the Project Scientist of Akatsuki. MN is the Project Manager and TI is the former Project Scientist of Akatsuki. MU was deeply involved in designing the PtSi CSD/CCD sensor for IR2.

\section{Author details}

${ }^{1}$ Institute of Space and Astronautical Science, Japan Aerospace Exploration Agency, 3-1-1 Yoshinodai, Chuo-ku, Sagamihara, Kanagawa 252-5210, Japan. 2 Department of Space and Astronautical Science, School of Physics, SOKENDAl, 3-1-1 Yoshinodai, Chuo-ku, Sagamihara, Kanagawa 252-5210, Japan. ${ }^{3}$ Department of Geophysics, Graduate School of Science, Tohoku University, Aramaki-aza-Aoba 6-3, Aoba, Sendai, Miyagi 980-8578, Japan. ${ }^{4}$ Department of Planetology, Center for Planetary Science, Kobe University, 7-1-48, Minamimachi, Minatojima, Chuo-ku, Kobe 650-0047, Japan. ${ }^{5}$ Department of Earth Science, Okayama University, 3-1-1 Tsushimanaka, Kita, Okayama 700-8530, Japan. ${ }^{6}$ Faculty of Environmental Earth Science, Hokkaido University, N10W5, Sapporo, Hokkaido 060-0810, Japan. ${ }^{7}$ Department of Complexity Science and Engineering, Graduate School of Frontier Sciences, The University of Tokyo, Kiban-tou 4H7, 5-1-5 Kashiwanoha, Kashiwa, Chiba 277-8561, Japan. ${ }^{8}$ FAST Corporation, 2791-5, Shimotsuruma, Yamato, Kanagawa, Japan. ${ }^{9}$ Graduate School of Natural Science and Technology, Okayama University, 3-1-1 Tsushimanaka, Kita, Okayama 700-8530, Japan. ${ }^{10}$ Faculty of Tourism, Wakayama University, Sakaedani 930, Wakayama City 640-8510, Japan. ${ }^{11}$ ARC-Space, CAIST, The University of Aizu, 90 Kami-lawase, Tsuruga, Ikki-machi, Aizu-Wakamatsu, Fukushima 965-8580, Japan. ${ }^{12}$ Planetary Exploration Research Center, Chiba Institute of Technology, 2-17-1 Tsudanuma, Narashino-shi, Chiba 275-0016, Japan. ${ }^{13}$ Department of Physics, Rikkyo University, 3-34-1 Nishi-Ikebukuro, Toshima-ku, Tokyo 171-8501, Japan. ${ }^{14}$ School of Engineering, The University of Shiga Prefecture, 2500 Hassaka-cho, Hikone, Shiga 522-8533, Japan. ${ }^{15}$ Faculty of Science, Kyoto Sangyo University, Motoyama, Kamigamo Kita-ku, Kyoto City 603-8555, Japan. ${ }^{16}$ Department of Information Engineering, National Institute of Technology, Matsue College, 14-4 Nishi-lkuma, Matsue, Shimane 690-8518, Japan. ${ }^{17}$ School of Commerce, Senshu University, 2-1-1, Higashimita, Tama-ku, Kawasaki-Shi, Kanagawa 214-8580, Japan. 


\section{Acknowledgements}

The authors are grateful to Prof. M. Kimata, Ritsumeikan University, for designing the PtSi detector. Dr. K. Uemizu, National Astronomical Observatory of Japan, played a key role in the development of the software to electronically drive the detector. Special thanks go to the manufacturers of IR2 (Sumitomo Heavy Industries, Ltd., for the system; Nikon for the optics; Magoshi for the baffle; and Fujitoku and Barr Associates for the filters). NEC developed the Akatsuki spacecraft system and has been supporting the operation since the launch. Comments and suggestions from two anonymous reviewers greatly improved the quality of the original manuscript and are appreciated.

\section{Competing interests}

The authors declare that they have no competing interests.

\section{Availability of data and materials}

Akatsuki data will be available from the Akatsuki Project as well as NASA's Planetary Data System starting in the summer of 2017 with regular additions of new data every 6 months.

\section{Ethics approval and consent to participate and consent to publish} Not applicable.

\section{Funding}

TS was supported by JSPS KAKENHI Grant Number $16 \mathrm{H} 05738$ to perform this study.

\section{Publisher's Note}

Springer Nature remains neutral with regard to jurisdictional claims in published maps and institutional affiliations.

Received: 29 June 2017 Accepted: 23 October 2017

Published online: 03 November 2017

\section{References}

Akiyama A et al (1994) 1040 × 1040 infrared charge sweep device imager with PtSi Schottky-barrier detectors. Opt Eng 33:64-71

Allen D, Crawford J (1984) Cloud structure on the dark side of Venus. Nature 307:222-224

Carlson RW et al (1993) Variations in Venus cloud particle properties: a new view of Venus's cloud morphology as observed by the Galileo near-infrared mapping spectrometer. Planet Space Sci 41:477-485

Drossart P et al (2007) A dynamic upper atmosphere of Venus as revealed by VIRTIS on Venus Express. Nature 450:641-645

Fukuhara T et al (2017) Large stationary gravity wave in the atmosphere of Venus. Nat Geosci 10:85-88. https://doi.org/10.1038/ngeo2873

García Muñoz A, Pérez-Hoyos S, Sánchez-Lavega A (2014) Glory revealed in disk-integrated photometry of Venus. Astron Astrophys 566(L1):1-6

Grinspoon DH et al (1993) Probing Venus's cloud structure with Galileo NIMS Planet Space Sci 41(7):515-542

Haus R, Kappel D, Arnold G (2015) Lower atmosphere minor gas abundances as retrieved from Venus Express VIRTIS-M-IR data at $2.3 \mu \mathrm{m}$. Planet Space Sci 105:159-174

Horinouchi T et al (2017a) Equatorial jet in the lower cloud layer of Venus revealed by Akatsuki. Nat Geosci 10:646-651. https://doi.org/10.1038/ ngeo3016

Horinouchi T, Murakami S, Kouyama T, Ogohara K, Yamazaki A, Yamada M, Watanabe S (2017b) Image velocimetry for clouds with relaxation labeling based on deformation consistency. Meas Sci Technol 28(8):085301. https://doi.org/10.1088/1361-6501/aa695c

Howell SB (2000) Handbook of CCD astronomy. Cambridge University Press, Cambridge
Hueso R, Peralta J, Sánchez-Lavega A (2012) Assessing the long-term variability of Venus winds at cloud level from VIRTIS-Venus Express. Icarus 217:585-598

Ignatiev NI et al (2009) Altimetry of the Venus cloud tops from the Venus Express observations. J Geophys Res 114:E00B43

Ikegawa S, Horinouchi T (2016) Improved automatic estimation of winds at the cloud top of Venus using superposition of cross-correlation surfaces. Icarus 271:98-119

Light-Curve Generator (1999) American Association of Variable Star Observers. https://www.aavso.org/lcg. Accessed Sep 2017

McGouldrick K, Baines KH, Momary TW, Grinspoon DH (2008) Venus Express/ VIRTIS observations of middle and lower cloud variability and implications for dynamics. J Geophys Res Planets 113:E00B14. doi:10.1029/200 8JE003113

Nakamura M et al (2011) Overview of Venus orbiter, Akatsuki. Earth Planets Space 63:443-457. https://doi.org/10.5047/eps.2011.02.009

Nakamura M et al (2016) AKATSUKI returns to Venus. Earth Planets Space 68:75 https://doi.org/10.1186/s40623-016-0457-6

Ogohara K et al(2017) Overview of Akatsuki data products: the first quality and accuracy estimations. Earth Planets Space, submitted to the Akatsuk special issue (in revision)

Peralta J, Lee YJ, McGouldrick K, Sagawa H, Sánchez-Lavega A, Imamura T, Widemann T, Nakamura M (2017) Overview of useful spectral regions for Venus: an update to encourage observations complementary to the Akatsuki mission. Icarus 288:235-239

Petrova EV, Shalygina OS, Markiewicz WJ (2015) The VMCNEx photometry at small phase angles: glory and the physical properties in the upper cloud layer of Venus. Planet Space Sci 113-114:120-134

Piccioni G et al (2007) South-polar features on Venus similar to those near the north pole. Nature 450:637-640

Satoh T et al (2015) Venus' clouds as inferred from the phase curves acquired by IR1 and IR2 on board Akatsuki. Icarus 248:213-220. https://doi. org/10.1016/j.icarus.2014.10.030

Satoh T et al (2016) Development and in-flight calibration of IR2: 2- $\mu$ m camera onboard Japan's Venus orbiter, Akatsuki. Earth Planets Space 68:74. https://doi.org/10.1186/s40623-016-0451-z

Svedhem H, Titov D, Taylor F, Witasse O (2009) Venus Express mission. J Geophys Res. https://doi.org/10.1029/2008JE003184

Taylor FW, Crisp D (1997) near-infrared sounding of the lower atmosphere of Venus. In: Bougher SW, Hunten DM, Phillips RJ (eds) Venus II: geology, geophysics, atmosphere, and solar wind environment. University of Arizona Press, Tucson, pp 325-351

Titov DV et al (2009) Venus Express: highlights of the nominal mission. Sol Syst Res 43:185-209. https://doi.org/10.1134/S0038094609030010

Two Micron All Sky Survey (2MASS) (2006) Infrared processing and analysis Center, Caltech, http://www.ipac.caltech.edu/2mass/. Accessed June 2016

Wigner EP (1997) On the constant A in Richardson's equation. In: Wightman AS (ed) Part I: physical chemistry. Part II: solid state physics. Springer, Berlin, pp 426-430. https://doi.org/10.1007/978-3-642-59033-7_41

\section{Submit your manuscript to a SpringerOpen ${ }^{\circ}$ journal and benefit from:}

- Convenient online submission

- Rigorous peer review

- Open access: articles freely available online

- High visibility within the field

Retaining the copyright to your article

Submit your next manuscript at springeropen.com 